\title{
RATE OF CONVERGENCE IN NONLINEAR HARTREE DYNAMICS WITH FACTORIZED INITIAL DATA
}

\author{
LI CHEN AND JI OON LEE
}

\begin{abstract}
The mean field dynamics of an $N$-particle weekly interacting Boson system can be described by the nonlinear Hartree equation. In this paper, we present estimates on the $1 / N$ rate of convergence of many-body Schrödinger dynamics to the one-body nonlinear Hartree dynamics with factorized initial data with two-body interaction potential $V$ in $L^{3}\left(\mathbb{R}^{3}\right)+L^{\infty}\left(\mathbb{R}^{3}\right)$.
\end{abstract}

\section{Introduction and Main Result}

1.1. Setting and history of the problem. In a non-relativistic case, the dynamics of an $N$-particle system is governed by the Schrödinger equation. For a real physical system, however, $N$ is usually very large so that it is hopeless to solve the $N$-body Schrödinger equation directly. There were many efforts to describe such a system by approximating by a simpler dynamics. One of the most important cases is a system of $N$-weakly interacting Bosons, which can be approximated well by using the nonlinear Hartree equation.

We consider a system of $N$-interacting three-dimensional Bosons in $\mathbb{R}^{3}$, described on $\left(L^{2}\left(\mathbb{R}^{3 N}\right)\right)_{s}$, the subspace of $L^{2}\left(\mathbb{R}^{3 N}, d X_{N}\right)$ consisting of all symmetric functions, where $X_{N}:=\left(x_{1}, x_{2}, \cdots, x_{N}\right)$. Given the two-particle interaction $V$, the mean-field Hamiltonian of this system is

$$
H_{N}:=-\sum_{j=1}^{N} \Delta_{j}+\frac{1}{N} \sum_{i<j}^{N} V\left(x_{i}-x_{j}\right),
$$

where $x_{i} \in \mathbb{R}^{3}$ are the positions of the particles and $\Delta_{i}$ denotes the Laplacian with respect to $i$-th particle. Note that the coupling constant $1 / N$ guarantees that the kinetic energy and the potential energy are typically of the same order.

We assume that the initial state is factorized, i.e.

$$
\psi\left(X_{N}\right)=\prod_{j=1}^{N} \varphi\left(x_{j}\right)
$$

for some $\varphi \in H^{1}\left(\mathbb{R}^{3}\right)$ with $\|\varphi\|_{L^{2}\left(\mathbb{R}^{3}\right)}=1$. Its time evolution, $\psi_{t}$, satisfies the $N$-body Schrödinger equation,

$$
i \partial_{t} \psi_{t}=H_{N} \psi_{t}
$$

with the initial data $\psi_{0}=\psi$.

It is well known in this system that the factorization is approximately preserved under the time evolution, and, in fact, we can expect that

$$
\psi_{t}\left(X_{N}\right) \simeq \prod_{j=1}^{N} \varphi_{t}\left(x_{j}\right)
$$

where $\varphi_{t}$ is given by the solution of the nonlinear Hartree equation,

$$
i \partial_{t} \varphi_{t}=-\Delta \varphi_{t}+\left(V *\left|\varphi_{t}\right|^{2}\right) \varphi_{t},\left.\quad \varphi_{t}\right|_{t=0}=\varphi .
$$

Li Chen is partially supported by National Natural Science Foundation of China (NSFC), grant number 10871112. 
To give a meaning to (1.4), we introduce marginal densities. The marginal density associated with $\psi_{t}$ is defined to be the orthogonal projection onto $\psi_{t}$, and we denote this by bracket notation,

$$
\gamma_{t}=\left|\psi_{t}\right\rangle\left\langle\psi_{t}\right|
$$

The kernel of $\gamma_{t}$ is given by

$$
\gamma_{t}\left(X_{N} ; X_{N}^{\prime}\right)=\psi_{t}\left(X_{N}\right) \overline{\psi_{t}}\left(X_{N}^{\prime}\right) .
$$

For $k=1, \cdots, N-1$, we also define $k$-particle marginal density $\gamma_{t}^{(k)}$ associated with $\psi_{t}$ by taking the partial trace of $\gamma_{t}$ over the last $(N-k)$ particles, i.e., the kernel of $\gamma_{t}^{(k)}$ is given by

$$
\gamma_{t}^{(k)}\left(X_{k} ; X_{k}^{\prime}\right)=\int d x_{k+1} \cdots d x_{N} \gamma_{t}\left(X_{k}, x_{k+1}, \cdots, x_{N} ; X_{k}^{\prime}, x_{k+1}, \cdots, x_{N}\right),
$$

where $X_{k}=\left(x_{1}, \cdots, x_{k}\right)$ and $X_{k}^{\prime}=\left(x_{1}^{\prime}, \cdots, x_{k}^{\prime}\right)$.

In terms of marginal densities, (1.2) is equivalent to

$$
\gamma=\gamma_{0}=|\varphi\rangle\left\langle\left.\varphi\right|^{\otimes N}\right.
$$

and it is expected to show that its time evolution satisfies the convergence

$$
\gamma_{t}^{(k)} \rightarrow\left|\varphi_{t}\right\rangle\left\langle\left.\varphi_{t}\right|^{\otimes k} \quad \text { as } N \rightarrow \infty\right.
$$

in the trace norm topology.

Spohn 11 proved (1.10), assuming the interaction potential $V$ is bounded, and Erdös and Yau 3 ] extended this result further to obtain a rigorous derivation of the Hartree equation for the Coulomb interaction case. These results were based on the study of dynamics of marginal densities, which is governed by BBGKY hierarchy

$$
\begin{aligned}
i \partial_{t} \gamma_{t}^{(k)}= & \sum_{j=1}^{k}\left[-\Delta_{j}, \gamma_{t}^{(k)}\right]+\frac{1}{N} \sum_{i<j}^{k}\left[V\left(x_{i}-x_{j}\right), \gamma_{t}^{(k)}\right] \\
& +\frac{N-k}{N} \sum_{j=1}^{k} \operatorname{Tr}_{k+1}\left[V\left(x_{j}-x_{k+1}\right), \gamma_{t}^{(k+1)}\right],
\end{aligned}
$$

where $\operatorname{Tr}_{k+1}$ denotes the partial trace over the $(k+1)$-st particle. In this method, compactness of the sequence of marginal densities $\left\{\gamma_{t}^{(k)}\right\}_{k=1}^{N}$ is first shown, then that any limit point of the sequence is a solution of the infinite hierarchy is proved, and finally, proving the uniqueness of the solution gives the desired result.

Another approach was introduced by Hepp [6] and extended by Ginibre and Velo [4, 5]. In this approach, the time evolution of coherent states was studied in the second quantized Fock-space representation. Using this method, the rate of convergence in (1.10) was proved by Rodnianski and Schlein [10, where they proved that, for factorized initial data and for the interaction potential such that $V^{2}(x) \leq C\left(1-\Delta_{x}\right)$

$$
\left.\operatorname{Tr}\left|\gamma_{t}^{(k)}-\right| \varphi_{t}\right\rangle\left\langle\left.\varphi_{t}\right|^{\otimes k}\right| \leq \frac{C e^{K t}}{\sqrt{N}}
$$

for some constants $C$ and $K$.

Knowles and Pickl 99 introduced a new method to prove (1.12) where the interaction potential $V \in L^{2}\left(\mathbb{R}^{3}\right)+L^{\infty}\left(\mathbb{R}^{3}\right)$. They also extended the result to more singular interaction potentials, where the rate of convergence becomes weaker as the singularity in the interaction potential stronger.

The optimal result for this quantitative estimate must be of the order $1 / N$, since the bound of the form

$$
\left.\operatorname{Tr}\left|\gamma_{t}^{(k)}-\right| \varphi_{t}\right\rangle\left\langle\left.\varphi_{t}\right|^{\otimes k}\right| \leq \frac{C e^{K t}}{N}
$$


is proved for the coherent case by Rodnianski and Schlein [10 and for the factorized initial state case with the bounded interaction potential by Erdös and Schlein [2].

In this paper, we first observe that the $\mathcal{U}_{2}$ dynamics, which was introduced by Ginibre and Velo [4, [5], gives the $O(1 / N)$ rate of convergence. The goal of this paper is to show that for factorized initial data, there exist constants $C$ and $K$ and $\|\varphi\|_{H^{1}}$ such that

$$
\left.\operatorname{Tr}\left|\gamma_{t}^{(k)}-\right| \varphi_{t}\right\rangle\left\langle\left.\varphi_{t}\right|^{\otimes k}\right| \leq \frac{C e^{K t}}{N}
$$

for $V \in L^{3}\left(\mathbb{R}^{3}\right)+L^{\infty}\left(\mathbb{R}^{3}\right)$.

1.2. Notations and tools to be used. Let $\mathcal{F}_{X}$ be the Fock space of symmetric functions, i.e.

$$
\mathcal{F}_{X}:=\bigoplus_{n \geq 0}\left(L^{2}\left(\mathbb{R}^{3 n}\right)\right)_{s}
$$

where $\left(L^{2}\left(\mathbb{R}^{0}\right)\right)_{s}=\mathbb{C}$. Here, $s$ denotes the subspace of symmetric functions. A vector $\psi$ in $\mathcal{F}_{X}$ is a sequence $\psi=\left\{\psi^{(n)}\right\}_{n \geq 0}$ of $n$-particle wavefunctions $\psi^{(n)} \in\left(L^{2}\left(\mathbb{R}^{3 n}\right)\right)_{s}$. The scalar product between $\psi_{a}, \psi_{b} \in \mathcal{F}_{X}$ is defined by

$$
\left\langle\psi_{a}, \psi_{b}\right\rangle_{\mathcal{F}_{X}}=\sum_{n \geq 0}\left\langle\psi_{a}^{(n)}, \psi_{b}^{(n)}\right\rangle_{L^{2}\left(\mathbb{R}^{3 n}\right)}
$$

and we will omit the subscript $\mathcal{F}_{X}$ from now on. We let

$$
\Omega:=\{1,0,0, \cdots\} \in \mathcal{F}_{X}
$$

which is called the vacuum. We will also make use of the space

$$
\mathcal{F}:=L^{\infty}\left([0, T], \mathcal{F}_{X}\right) .
$$

For $n \geq 1$, let

$$
F_{n}:=\left\{\xi_{n}\left(t, X_{n}\right) \mid \xi_{n} \in L_{t}^{\infty}\left(L_{X_{n}}^{2}\right)_{s} \cap \bigcap_{i<j}^{n} L_{t}^{2} L_{x_{i}-x_{j}}^{6} L_{x_{i}+x_{j}}^{2} L_{X_{n} \backslash\left\{x_{i}, x_{j}\right\}}^{2}\right\}
$$

with

$$
\left\|\xi_{n}\right\|_{F_{n}}=\left\|\xi_{n}\left(t, X_{n}\right)\right\|_{L_{t}^{\infty} L_{X_{n}}^{2}}+\max _{1 \leq i<j \leq n}\left\{\left\|\xi_{n}\left(t, X_{n}\right)\right\|_{L_{t}^{2} L_{x_{i}-x_{j}}^{6} L_{x_{i}+x_{j}}^{2} L_{X_{n} \backslash\left\{x_{i}, x_{j}\right\}}^{2}}\right\},
$$

and let $F_{0}=\mathbb{C}$. Here, we again consider a fixed time interval $t \in[0, T]$ only. We define another space

$$
\widetilde{\mathcal{F}}:=\bigoplus_{n \geq 0} F_{n}
$$

equipped with the norm

$$
\|\cdot\|_{\widetilde{\mathcal{F}}}^{2}=\sum_{n \geq 0}\|\cdot\|_{F_{n}}^{2} .
$$

On $\mathcal{F}_{X}$, the creation operator $a_{x}^{\dagger}$ and the annihilation operator $a_{x}$ for $x \in \mathbb{R}^{3}$ are defined by

$$
\begin{aligned}
\left(a_{x}^{\dagger} \psi\right)^{(n)}\left(x_{1}, \cdots, x_{n}\right) & =\frac{1}{\sqrt{n}} \sum_{j=1}^{n} \delta\left(x-x_{j}\right) \psi^{(n-1)}\left(x_{1}, \cdots, x_{j-1}, x_{j+1}, \cdots, x_{n}\right), \\
\left(a_{x} \psi\right)^{(n)}\left(x_{1}, \cdots, x_{n}\right) & =\sqrt{n+1} \psi^{(n+1)}\left(x, x_{1}, \cdots, x_{n}\right) .
\end{aligned}
$$

For $f \in L^{2}\left(\mathbb{R}^{3}\right), a^{\dagger}(f)$ and $a(f)$ are given by

$$
\begin{aligned}
a^{\dagger}(f) & =\int d x f(x) a_{x}^{\dagger}, \\
a(f) & =\int d x \overline{f(x)} a_{x} .
\end{aligned}
$$


The creation operator $a^{\dagger}(f)$ is the adjoint of the annihilation operator $a(f)$, and they satisfy the canonical commutation relations

$$
\left[a(f), a^{\dagger}(g)\right]=\langle f, g\rangle_{L^{2}\left(\mathbb{R}^{3}\right)}, \quad[a(f), a(g)]=\left[a^{\dagger}(f), a^{\dagger}(g)\right]=0 .
$$

The number operator $\mathcal{N}$ on $\mathcal{F}_{X}$ is defined by

$$
\mathcal{N}:=\int d x a_{x}^{\dagger} a_{x}
$$

and it also satisfies

$$
(\mathcal{N} \psi)^{(n)}=n \psi^{(n)} \text {. }
$$

We define the Hamiltonian $\mathcal{H}_{N}$ on $\mathcal{F}_{X}$ by $\left(\mathcal{H}_{N} \psi\right)^{(n)}=H_{n} \psi^{(n)}$. Using the operators $a_{x}$ and $a_{x}^{\dagger}$, it can be rewritten as

$$
\mathcal{H}_{N}=\int d x a_{x}^{\dagger}\left(-\Delta_{x}\right) a_{x}+\frac{1}{2 N} \iint d x d y V(x-y) a_{x}^{\dagger} a_{y}^{\dagger} a_{y} a_{x} .
$$

For $f \in L^{2}\left(\mathbb{R}^{3}\right)$, the Weyl operator $W(f)$ and the coherent state $\psi(f)$ are given by

$$
W(f):=\exp \left(a^{\dagger}(f)-a(f)\right)
$$

and

The Weyl operator $W(f)$ satisfies

$$
\psi(f):=W(f) \Omega .
$$

$$
W(f)=e^{-\|f\|^{2} / 2} \exp \left(a^{\dagger}(f)\right) \exp (-a(f)) .
$$

Important properties of Weyl operators are collected in Lemma 2.2 of [10].

1.3. Main theorem. In this paper, for factorized initial data and the interaction potentials $V \in$ $L^{3}\left(\mathbb{R}^{3}\right)+L^{\infty}\left(\mathbb{R}^{3}\right)$, we extend the convergence rate $O\left(1 / N^{\frac{1}{2}}\right)$ in Rodnianski and Schlein [10] to $O(1 / N)$. More precisely, we prove the following theorem.

Theorem 1.1. Suppose that $V=V_{1}+V_{2}$ where $V_{1} \in L^{3}\left(\mathbb{R}^{3}\right)$ and $V_{2} \in L^{\infty}\left(\mathbb{R}^{3}\right)$. Let $\gamma_{N, t}^{(1)}$ be the one-particle marginal density associated with the time evolution of the factorized initial state $\left\{0, \cdots, 0, \varphi^{\otimes N}, 0, \cdots\right\}$ as in (1.8). Then there exist constants $C$ and $K$, depending only on $\|\varphi\|_{H^{1}}$, $\left\|V_{1}\right\|_{L^{3}}$, and $\left\|V_{2}\right\|_{L^{\infty}}$ such that

$$
\left.\operatorname{Tr}\left|\gamma_{N, t}^{(1)}-\right| \varphi_{t}\right\rangle\left\langle\varphi_{t}|| \leq \frac{C e^{K t}}{N}\right.
$$

Remark 1. Using the same arguments used to prove (1.34), we can extend the result to the higher marginals $\gamma_{N, t}^{(k)}$.

Remark 2. For the proof of Theorem [1.1, we first assume that $t \in[0, T]$ and prove the bound

$$
\left.\operatorname{Tr}\left|\gamma_{N, t}^{(1)}-\right| \varphi_{t}\right\rangle\left\langle\varphi_{t}|| \leq \frac{C e^{K T}}{N} .\right.
$$

Letting $T=t$, Theorem 1.1 follows.

Remark 3. What we actually prove in this paper is

$$
\left.\left|\gamma_{N, t}^{(1)}-\right| \varphi_{t}\right\rangle\left\langle\varphi_{t}||_{H S} \leq \frac{C e^{K t}}{N},\right.
$$

which implies (1.34). Here, $\|\cdot\|_{H S}$ denotes the Hilbert-Schmidt norm. The argument for this implication can be found in Remark 1.4 of [10].

Remark 4. Note that the nonlinear Hartree equation (1.5) is globally well-posed when $V$ satisfies the assumptions of Theorem 1.1. See Remark 1.3 of [10] for further detail. 
In [10, the authors had proved the convergence rate is $1 / N$ with initial data is coherent state. Then, by writing the factorized state

$$
\left\{0, \cdots, 0, \varphi^{\otimes N}, 0, \cdots\right\}=\frac{\left(a^{\dagger}(\varphi)\right)^{N}}{\sqrt{N !}} \Omega=d_{N} \int_{0}^{2 \pi} \frac{d \theta}{2 \pi} e^{i \theta N} W\left(e^{-i \theta \sqrt{N} \varphi}\right) \Omega
$$

with the constant

$$
d_{N}:=\frac{\sqrt{N !}}{N^{N / 2} e^{-N / 2}} \simeq N^{1 / 4}
$$

they could prove that the rate of convergence is of order $1 / \sqrt{N}$.

To explain our idea more explicitly, we need first to give a brief outline of the proof of the main theorem in [10].

1.4. Outline of the idea in [10]. Let $\Gamma_{N, t}^{(1)}(x, y)$ be the kernel of the one particle marginal density associated with the time evolution of the coherent state $W(\sqrt{N} \varphi) \Omega$, which is defined by

$$
\Gamma_{N, t}^{(1)}(x, y)=\frac{1}{N}\left\langle e^{-i \mathcal{H}_{N} t} W(\sqrt{N} \varphi) \Omega, a_{y}^{\dagger} a_{x} e^{-i \mathcal{H}_{N} t} W(\sqrt{N} \varphi) \Omega\right\rangle,
$$

We expect that the limit of the kernel of one particle marginal density is $\bar{\varphi}_{t}(x) \varphi_{t}(y)$, thus we expand $\Gamma_{N, t}^{(1)}(x, y)$ in terms of $\left(a_{x}-\sqrt{N} \varphi_{t}(x)\right)$ and $\left(a_{y}^{\dagger}-\sqrt{N} \overline{\varphi_{t}}(y)\right)$. Then, we get

$$
\begin{aligned}
\Gamma_{N, t}^{(1)}(x, y)= & \varphi_{t}(x) \overline{\varphi_{t}}(y) \\
& +\frac{1}{N}\left\langle\Omega, W^{\dagger}(\sqrt{N} \varphi) e^{i \mathcal{H}_{N} t}\left(a_{y}^{\dagger}-\sqrt{N} \overline{\varphi_{t}}(y)\right)\left(a_{x}-\sqrt{N} \varphi_{t}(x)\right) e^{-i \mathcal{H}_{N} t} W(\sqrt{N} \varphi) \Omega\right\rangle \\
& +\frac{\varphi_{t}(x)}{\sqrt{N}}\left\langle\Omega, W^{\dagger}(\sqrt{N} \varphi) e^{i \mathcal{H}_{N} t}\left(a_{y}^{\dagger}-\sqrt{N} \overline{\varphi_{t}}(y)\right) e^{-i \mathcal{H}_{N} t} W(\sqrt{N} \varphi) \Omega\right\rangle \\
& +\frac{\overline{\varphi_{t}}(y)}{\sqrt{N}}\left\langle\Omega, W^{\dagger}(\sqrt{N} \varphi) e^{i \mathcal{H}_{N} t}\left(a_{x}-\sqrt{N} \varphi_{t}(x)\right) e^{-i \mathcal{H}_{N} t} W(\sqrt{N} \varphi) \Omega\right\rangle .
\end{aligned}
$$

It was shown by Hepp [6] (also by Ginibre and Velo [4, 5]) that

$$
W^{\dagger}\left(\sqrt{N} \varphi_{s}\right) e^{i \mathcal{H}_{N}(t-s)}\left(a_{x}-\sqrt{N} \varphi_{t}(x)\right) e^{-i \mathcal{H}_{N}(t-s)} W\left(\sqrt{N} \varphi_{s}\right)=\mathcal{U}^{\dagger}(t ; s) a_{x} \mathcal{U}(t ; s),
$$

where $\mathcal{U}(t ; s)$ is a unitary operator defined through

$$
\mathcal{U}(t ; s):=W^{\dagger}\left(\sqrt{N} \varphi_{t}\right) e^{-i \mathcal{H} N(t-s)} W\left(\sqrt{N} \varphi_{s}\right) .
$$

The operator $\mathcal{U}(t ; s)$ also satisfies

$$
i \partial_{t} \mathcal{U}(t ; s)=\left(H_{2}(t)+H_{3}(t)+H_{4}\right) \mathcal{U}(t ; s), \quad \mathcal{U}(s ; s)=I,
$$

where the generators $H_{2}, H_{3}$, and $H_{4}$ are defined as follows:

$$
\begin{aligned}
H_{2}(t):= & \int d x \nabla_{x} a_{x}^{\dagger} \nabla_{x} a_{x}+\int d x\left(V *\left|\varphi_{t}\right|^{2}\right)(x) a_{x}^{\dagger} a_{x}+\iint d x d y V(x-y) \overline{\varphi_{t}}(x) \varphi_{t}(y) a_{y}^{\dagger} a_{x} \\
& +\frac{1}{2} \iint d x d y V(x-y)\left(\varphi_{t}(x) \varphi_{t}(y) a_{x}^{\dagger} a_{y}^{\dagger}+\overline{\varphi_{t}}(x) \overline{\varphi_{t}}(y) a_{x} a_{y}\right), \\
H_{3}(t):= & \frac{1}{\sqrt{N}} \iint d x d y V(x-y) \varphi_{t}(y) a_{x}^{\dagger} a_{y}^{\dagger} a_{x}+\frac{1}{\sqrt{N}} \iint d x d y V(x-y) \overline{\varphi_{t}}(y) a_{x}^{\dagger} a_{y} a_{x}, \\
H_{4}:= & \frac{1}{2 N} \iint d x d y V(x-y) a_{x}^{\dagger} a_{y}^{\dagger} a_{x} a_{y} .
\end{aligned}
$$


Using $\mathcal{U}(t ; s)$, we can rewrite (1.40) as

$$
\begin{aligned}
& \Gamma_{N, t}^{(1)}(x, y)-\varphi_{t}(x) \overline{\varphi_{t}}(y) \\
& =\frac{1}{N}\left\langle\Omega, \mathcal{U}^{\dagger}(t ; 0) a_{y}^{\dagger} a_{x} \mathcal{U}(t ; 0) \Omega\right\rangle \\
& \quad+\frac{\varphi_{t}(x)}{\sqrt{N}}\left\langle\Omega, \mathcal{U}^{\dagger}(t ; 0) a_{y}^{\dagger} \mathcal{U}(t ; 0) \Omega\right\rangle+\frac{\overline{\varphi_{t}}(y)}{\sqrt{N}}\left\langle\Omega, \mathcal{U}^{\dagger}(t ; 0) a_{x} \mathcal{U}(t ; 0) \Omega\right\rangle .
\end{aligned}
$$

Proposition 4.1 of [4] shows that there exists a unique group of unitary operators $\widetilde{\mathcal{U}}_{2}(t ; s)$ satisfying

$$
i \partial_{t} \widetilde{\mathcal{U}}_{2}(t ; s)=\left(\widetilde{H}_{2}(t)-H_{0}\right) \widetilde{\mathcal{U}}_{2}(t ; s), \quad \widetilde{\mathcal{U}}_{2}(s ; s)=I,
$$

where $\widetilde{H}_{2}(t)$ is defined by

We define

$$
\widetilde{H}_{2}(t):=e^{i H_{0} t} H_{2}(t) e^{-i H_{0} t}
$$

$$
\mathcal{U}_{2}(t ; s):=e^{-i H_{0} t} \widetilde{\mathcal{U}}_{2}(t ; s) e^{i H_{0} s} .
$$

We note that $\mathcal{U}_{2}$ is well-defined on $\mathcal{F}_{X}$, since the free evolutions $e^{-i H_{0} t}$ and $e^{i H_{0} s}$ are well-defined on $\mathcal{F}_{X}$, and the unitary operator $\widetilde{\mathcal{U}}_{2}(t ; s)$ is also well-defined on $\mathcal{F}_{X}$. Since the operators $\widetilde{\mathcal{U}}_{2}, e^{-i H_{0} t}$, and $e^{i H_{0} s}$ are bounded in $\mathcal{D}\left(\mathcal{N}^{\delta}\right)$ for any $\delta \in \mathbb{R}$, so is $\mathcal{U}_{2}$.

Furthermore, it can be proved from Proposition 2.2 of [5] and from Lemma 7.1 that $\mathcal{U}_{2}$ is strongly differentiable from $\mathcal{Q}\left(H_{0}+\mathcal{N}\right)$, the form domain of $H_{0}+\mathcal{N}$, to its dual $\mathcal{Q}^{*}\left(H_{0}+\mathcal{N}\right)$. Thus, on a dense subset $\mathcal{D}\left(H_{0}\right) \cap \mathcal{D}(\mathcal{N})$ of $\mathcal{F}_{X}$, we can check from the definition that

$$
i \partial_{t} \mathcal{U}_{2}(t ; s)=H_{2}(t) \mathcal{U}_{2}(t ; s), \quad \mathcal{U}_{2}(s ; s)=I .
$$

For simplicity, we will use notations

$$
\mathcal{U}(t):=\mathcal{U}(t ; 0), \quad \mathcal{U}_{2}(t):=\mathcal{U}_{2}(t ; 0)
$$

To estimate the difference between $\Gamma_{N, t}^{(1)}(x, y)$ and $\varphi_{t}(x) \overline{\varphi_{t}}(y)$ as in (1.47), one needs to find a good approximation of $\mathcal{U}(t ; 0)$, which conserves the parity of the number of particles, and we call it $\tilde{\mathcal{U}}(t ; 0)$. Then

$$
\begin{aligned}
\Gamma_{N, t}^{(1)}(x, y)-\varphi_{t}(x) \overline{\varphi_{t}}(y) \\
=\frac{1}{N}\left\langle\Omega, \mathcal{U}^{\dagger}(t ; 0) a_{y}^{\dagger} a_{x} \mathcal{U}(t ; 0) \Omega\right\rangle \\
\quad+\frac{\varphi_{t}(x)}{\sqrt{N}}\left(\left\langle\Omega, \mathcal{U}^{\dagger}(t ; 0) a_{y}^{\dagger}(\mathcal{U}(t ; 0)-\widetilde{\mathcal{U}}(t ; 0)) \Omega\right\rangle+\left\langle\Omega,\left(\mathcal{U}^{\dagger}(t ; 0)-\widetilde{\mathcal{U}}^{\dagger}(t ; 0)\right) a_{y}^{\dagger} \widetilde{\mathcal{U}}(t ; 0) \Omega\right\rangle\right) \\
\quad+\frac{\frac{\overline{\varphi_{t}}(y)}{\sqrt{N}}\left(\left\langle\Omega, \mathcal{U}^{\dagger}(t ; 0) a_{x}(\mathcal{U}(t ; 0)-\widetilde{\mathcal{U}}(t ; 0)) \Omega\right\rangle+\left\langle\Omega,\left(\mathcal{U}^{\dagger}(t ; 0)-\widetilde{\mathcal{U}}^{\dagger}(t ; 0)\right) a_{x} \widetilde{\mathcal{U}}(t ; 0) \Omega\right\rangle\right) .}{}
\end{aligned}
$$

If we define $\tilde{\mathcal{U}}$ through

$$
i \partial_{t} \widetilde{\mathcal{U}}(t ; 0)=\left(H_{2}+H_{4}\right) \widetilde{\mathcal{U}}(t ; 0), \quad \widetilde{\mathcal{U}}(0 ; 0)=I,
$$

then it can be proved that

$$
\|\mathcal{U}(t ; 0) \Omega-\widetilde{\mathcal{U}}(t ; 0) \Omega\| \leq \frac{C e^{K t}}{\sqrt{N}}
$$

and

$$
\langle\mathcal{U}(t ; 0) \Omega, \mathcal{N} \mathcal{U}(t ; 0) \Omega\rangle \leq C e^{K t}
$$

which are Lemma 3.9 and Proposition 3.3 of [10], respectively. Thus,

$$
\left.\operatorname{Tr}\left|\Gamma_{N, t}^{(1)}-\right| \varphi_{t}\right\rangle\left\langle\varphi_{t}|| \leq \frac{C e^{K t}}{N} .\right.
$$


RATE OF CONVERGENCE IN NONLINEAR HARTREE DYNAMICS WITH FACTORIZED INITIAL DATA 7

Note that the results in [10] are under the assumption that $|V(x)|^{2} \leq D\left(1-\Delta_{x}\right)$ for some constant $D$. In this paper, we will use these estimates, since the potential $V$ in Theorem 1.1 satisfies the same assumption.

1.5. Factorized initial data. Recall that

$$
W^{\dagger}(\sqrt{N} \varphi) e^{i \mathcal{H}_{N} t}\left(a_{x}-\sqrt{N} \varphi_{t}(x)\right) e^{-i \mathcal{H}_{N} t} W(\sqrt{N} \varphi)=\mathcal{U}^{\dagger}(t) a_{x} \mathcal{U}(t)
$$

By definition, we have that

$$
(W(\sqrt{N} \varphi) \Omega)^{(N)}=e^{-N / 2} \frac{\left(a^{\dagger}(\sqrt{N} \varphi)\right)^{N}}{N !} \Omega=\frac{1}{d_{N}} \frac{\left(a^{\dagger}(\varphi)\right)^{N}}{\sqrt{N !}} \Omega .
$$

For factorized initial data, it follows from (1.58) and (1.59) that

$$
\begin{aligned}
& \gamma_{N, t}^{(1)}(x ; y)=\frac{1}{N}\left\langle\frac{\left(a^{\dagger}(\varphi)\right)^{N}}{\sqrt{N !}} \Omega, e^{i \mathcal{H}_{N} t} a_{y}^{\dagger} a_{x} e^{-i \mathcal{H}_{N} t} \frac{\left(a^{\dagger}(\varphi)\right)^{N}}{\sqrt{N !}} \Omega\right\rangle \\
& =\frac{d_{N}^{2}}{N}\left\langle e^{i \mathcal{H}_{N} t} a_{y} e^{-i \mathcal{H}_{N} t}(W(\sqrt{N} \varphi) \Omega)^{(N)}, e^{i \mathcal{H}_{N} t} a_{x} e^{-i \mathcal{H}_{N} t}(W(\sqrt{N} \varphi) \Omega)^{(N)}\right\rangle \\
& =\frac{d_{N}^{2}}{N}\left\langle\left(e^{i \mathcal{H}_{N} t} a_{y} e^{-i \mathcal{H}_{N} t} W(\sqrt{N} \varphi) \Omega\right)^{(N-1)},\left(e^{i \mathcal{H}_{N} t} a_{x} e^{-i \mathcal{H}_{N} t} W(\sqrt{N} \varphi) \Omega\right)^{(N-1)}\right\rangle \\
& =\frac{d_{N}^{2}}{N}\left\langle\left(W(\sqrt{N} \varphi) \mathcal{U}^{\dagger}(t)\left(a_{y}+\sqrt{N} \varphi_{t}(y)\right) \mathcal{U}(t) \Omega\right)^{(N-1)},\right. \\
& \left.\quad\left(W(\sqrt{N} \varphi) \mathcal{U}^{\dagger}(t)\left(a_{x}+\sqrt{N} \varphi_{t}(x)\right) \mathcal{U}(t) \Omega\right)^{(N-1)}\right\rangle .
\end{aligned}
$$

Thus, we obtain the following equation for one-particle marginal.

$$
\begin{aligned}
& \gamma_{N, t}^{(1)}(x ; y)-\bar{\varphi}_{t}(y) \varphi_{t}(x) \\
= & \frac{d_{N}^{2}}{N}\left\langle\left(W(\sqrt{N} \varphi) \mathcal{U}^{\dagger}(t) a_{y} \mathcal{U}(t) \Omega\right)^{(N-1)},\left(W(\sqrt{N} \varphi) \mathcal{U}^{\dagger}(t) a_{x} \mathcal{U}(t) \Omega\right)^{(N-1)}\right\rangle \\
& +\bar{\varphi}_{t}(y) \frac{d_{N}^{2}}{\sqrt{N}}\left\langle(W(\sqrt{N} \varphi) \Omega)^{(N-1)},\left(W(\sqrt{N} \varphi) \mathcal{U}^{\dagger}(t) a_{x} \mathcal{U}(t) \Omega\right)^{(N-1)}\right\rangle \\
& +\varphi_{t}(x) \frac{d_{N}^{2}}{\sqrt{N}}\left\langle\left(W(\sqrt{N} \varphi) \mathcal{U}^{\dagger}(t) a_{y} \mathcal{U}(t) \Omega\right)^{(N-1)},(W(\sqrt{N} \varphi) \Omega)^{(N-1)}\right\rangle .
\end{aligned}
$$

Define

$$
E(t, x, y):=\gamma_{N, t}^{(1)}(x ; y)-\bar{\varphi}_{t}(y) \varphi_{t}(x)
$$

We also define $E_{2}(t, x, y)$ by putting $\mathcal{U}_{2}$ instead of $\mathcal{U}$ in (1.61) as follows.

$$
\begin{aligned}
E_{2}(t, x, y)= & \frac{d_{N}^{2}}{N}\left\langle\left(W(\sqrt{N} \varphi) \mathcal{U}_{2}^{\dagger}(t) a_{y} \mathcal{U}_{2}(t) \Omega\right)^{(N-1)},\left(W(\sqrt{N} \varphi) \mathcal{U}_{2}^{\dagger}(t) a_{x} \mathcal{U}_{2}(t) \Omega\right)^{(N-1)}\right\rangle \\
& +\bar{\varphi}_{t}(y) \frac{d_{N}^{2}}{\sqrt{N}}\left\langle(W(\sqrt{N} \varphi) \Omega)^{(N-1)},\left(W(\sqrt{N} \varphi) \mathcal{U}_{2}^{\dagger}(t) a_{x} \mathcal{U}_{2}(t) \Omega\right)^{(N-1)}\right\rangle \\
& +\varphi_{t}(x) \frac{d_{N}^{2}}{\sqrt{N}}\left\langle\left(W(\sqrt{N} \varphi) \mathcal{U}_{2}^{\dagger}(t) a_{y} \mathcal{U}_{2}(t) \Omega\right)^{(N-1)},(W(\sqrt{N} \varphi) \Omega)^{(N-1)}\right\rangle .
\end{aligned}
$$


1.6. Arrangement of the paper. This paper is organized as follows:

(1) We will first study the evolution of $\mathcal{U}_{2}^{\dagger}(t) a_{x} \mathcal{U}_{2}(t)$ in section 2 , then, by using it, we will prove the following proposition.

Proposition 1.1. Let $E_{2}(t, x, y)$ be defined by (1.63). Then, there exist constants $C$ and $K$ such that

$$
\left\|E_{2}(t, x, y)\right\|_{L_{x, y}^{2}} \leq \frac{C e^{K t}}{N} .
$$

(2) To estimate the difference $\mathcal{U}(t)-\mathcal{U}_{2}(t)$, we study more regularity for $\mathcal{U}_{2}(t)$ dynamics in section 3. More precisely, we will prove the following proposition.

Proposition 1.2. Let $\psi_{2}(t):=\mathcal{U}_{2}(t ; s) \psi$ be the unique solution of initial value problem

$$
\left\{\begin{array}{l}
i \partial_{t}\left(\mathcal{N}^{j} \psi_{2}(t)\right)=\mathcal{N}^{j} H_{2}(t) \psi_{2}(t) \\
\psi_{2}(s)=\psi \in \mathcal{F}_{X}
\end{array} .\right.
$$

Then there exists a constant $C$ depending only on $j,\left\|V_{1}\right\|_{L^{3}},\left\|V_{2}\right\|_{L^{\infty}}$ and $\|\varphi\|_{H^{1}}$, such that

$$
\left\|\mathcal{N}^{j} \psi_{2}\right\|_{\widetilde{\mathcal{F}}} \leq C\left\|\mathcal{N}^{j} \psi\right\|+C T\left\|(\mathcal{N}+2)^{j+1} \psi_{2}\right\|_{\mathcal{F}}
$$

(3) Let

$$
\mathcal{R}_{y}(t):=\mathcal{U}^{\dagger}(t) a_{y} \mathcal{U}(t)-\mathcal{U}_{2}^{\dagger}(t) a_{y} \mathcal{U}_{2}(t)
$$

In Section 4, using Proposition 1.2 we prove the following proposition, which gives an estimate for the difference between $\mathcal{U}(t)$ dynamics and $\mathcal{U}_{2}(t)$ dynamics.

Proposition 1.3. Let $\mathcal{R}_{y}(t)$ be defined as in (1.67). Then, for all $j \in \mathbb{N}$, there exist constants $C$ and $K$ such that

$$
\left(\int d y\left\|(\mathcal{N}+1)^{\frac{j}{2}} \mathcal{R}_{y}(t) \Omega\right\|^{2}\right)^{\frac{1}{2}} \leq \frac{C e^{K T}}{\sqrt{N}} .
$$

(4) In Section 5, we prove the main theorem, using Proposition 1.1 Proposition 1.2, and Proposition 1.3. Some technical estimates are proved in Section 6.

\section{Evolution of $\mathcal{U}_{2}^{\dagger}(t) a_{x} \mathcal{U}_{2}(t)$}

2.1. Properties of $\mathcal{U}_{2}^{\dagger}(t) a_{x} \mathcal{U}_{2}(t)$. We first want to obtain some algebraic properties of an operator $\mathcal{U}_{2}^{\dagger}(t) a_{x} \mathcal{U}_{2}(t)$, which will act on the vacuum $\Omega$. For simplicity, we let

$$
\begin{aligned}
& a_{x}(t):=\mathcal{U}_{2}^{\dagger}(t) a_{x} \mathcal{U}_{2}(t), \\
& a_{x}^{\dagger}(t):=\mathcal{U}_{2}^{\dagger}(t) a_{x}^{\dagger} \mathcal{U}_{2}(t) .
\end{aligned}
$$

Formal calculation shows that the time derivatives of $a_{x}(t)$ and $a_{x}^{\dagger}(t)$ are given by

$$
i \partial_{t}\left(\begin{array}{c}
a_{x}(t) \\
a_{x}^{\dagger}(t)
\end{array}\right)=\mathcal{U}_{2}^{\dagger}(t)\left[\left(\begin{array}{c}
a_{x} \\
a_{x}^{\dagger}
\end{array}\right), H_{2}\right] \mathcal{U}_{2}(t)
$$

Commutators in the right hand side of (2.3) can be calculated explicitly as follows.

$$
\begin{gathered}
{\left[a_{x}, \int d y \nabla_{y} a_{y}^{\dagger} \nabla_{y} a_{y}\right]=-\Delta_{x} a_{x},} \\
{\left[a_{x}^{\dagger}, \int d y \nabla_{y} a_{y}^{\dagger} \nabla_{y} a_{y}\right]=\Delta_{x} a_{x}^{\dagger},} \\
{\left[a_{x}, \int d y\left(V *\left|\varphi_{t}\right|^{2}\right)(y) a_{y}^{\dagger} a_{y}\right]=\left(V *\left|\varphi_{t}\right|^{2}\right)(x) a_{x},} \\
{\left[a_{x}^{\dagger}, \int d y\left(V *\left|\varphi_{t}\right|^{2}\right)(y) a_{y}^{\dagger} a_{y}\right]=-\left(V *\left|\varphi_{t}\right|^{2}\right)(x) a_{x}^{\dagger},}
\end{gathered}
$$




$$
\begin{gathered}
{\left[a_{x}, \iint d y d z V(y-z) \overline{\varphi_{t}}(y) \varphi_{t}(z) a_{z}^{\dagger} a_{y}\right]=\int d y V(x-y) \overline{\varphi_{t}}(y) \varphi_{t}(x) a_{y},} \\
{\left[a_{x}^{\dagger}, \iint d y d z V(y-z) \overline{\varphi_{t}}(y) \varphi_{t}(z) a_{z}^{\dagger} a_{y}\right]=-\int d y V(x-y) \overline{\varphi_{t}}(x) \varphi_{t}(y) a_{y}^{\dagger},} \\
{\left[a_{x}, \frac{1}{2} \iint d y d z V(y-z) \varphi_{t}(y) \varphi_{t}(z) a_{y}^{\dagger} a_{z}^{\dagger}\right]=\int d y V(x-y) \varphi_{t}(x) \varphi_{t}(y) a_{y}^{\dagger},} \\
{\left[a_{x}^{\dagger}, \frac{1}{2} \iint d y d z V(y-z) \overline{\varphi_{t}}(y) \overline{\varphi_{t}}(z) a_{y} a_{z}\right]=-\int d y V(x-y) \overline{\varphi_{t}}(x) \overline{\varphi_{t}}(y) a_{y}}
\end{gathered}
$$

Now, (2.3) becomes

$$
\begin{aligned}
& i \partial_{t}\left(\begin{array}{c}
a_{x}(t) \\
a_{x}^{\dagger}(t)
\end{array}\right) \\
& =\mathcal{U}_{2}^{\dagger}(t)\left(\begin{array}{c}
-\Delta_{x} a_{x}+\left(V *\left|\varphi_{t}\right|^{2}\right)(x) a_{x} \\
\Delta_{x} a_{x}^{\dagger}-\left(V *\left|\varphi_{t}\right|^{2}\right)(x) a_{x}^{\dagger}
\end{array}\right) \mathcal{U}_{2}(t) \\
& +\mathcal{U}_{2}^{\dagger}(t)\left(\begin{array}{c}
\int d y V(x-y) \overline{\varphi_{t}}(y) \varphi_{t}(x) a_{y}+\int d y V(x-y) \varphi_{t}(x) \varphi_{t}(y) a_{y}^{\dagger} \\
-\int d y V(x-y) \overline{\varphi_{t}}(x) \varphi_{t}(y) a_{y}^{\dagger}-\int d y V(x-y) \overline{\varphi_{t}}(x) \overline{\varphi_{t}}(y) a_{y}
\end{array}\right) \mathcal{U}_{2}(t) \\
& =\left(\begin{array}{cc}
-\Delta_{x}+\left(V *\left|\varphi_{t}\right|^{2}\right)(x) & 0 \\
0 & \Delta_{x}-\left(V *\left|\varphi_{t}\right|^{2}\right)(x)
\end{array}\right)\left(\begin{array}{c}
a_{x}(t) \\
a_{x}^{\dagger}(t)
\end{array}\right) \\
& +\int d y\left(\begin{array}{cc}
V(x-y) \overline{\varphi_{t}}(y) \varphi_{t}(x) & V(x-y) \varphi_{t}(x) \varphi_{t}(y) \\
-V(x-y) \overline{\varphi_{t}}(x) \overline{\varphi_{t}}(y) & -V(x-y) \overline{\varphi_{t}}(x) \varphi_{t}(y)
\end{array}\right)\left(\begin{array}{c}
a_{y}(t) \\
a_{y}^{\dagger}(t)
\end{array}\right) .
\end{aligned}
$$

Using (2.4), we can immediately see that

$$
\left(\begin{array}{c}
a_{x}(t) \\
a_{x}^{\dagger}(t)
\end{array}\right)=G(t)\left(\begin{array}{c}
a_{x} \\
a_{x}^{\dagger}
\end{array}\right):=\left(\begin{array}{cc}
G_{1} & G_{2} \\
G_{3} & G_{4}
\end{array}\right)\left(\begin{array}{c}
a_{x} \\
a_{x}^{\dagger}
\end{array}\right) .
$$

Here, $G_{1}, G_{2}, G_{3}$, and $G_{4}$ depend on $t$, but we omitted it. Since

$$
\left(\mathcal{U}_{2}^{\dagger}(t) a_{x} \mathcal{U}_{2}(t)\right)^{\dagger}=\mathcal{U}_{2}^{\dagger}(t) a_{x}^{\dagger} \mathcal{U}_{2}(t)
$$

we can easily see that $G_{4}=\overline{G_{1}}$ and $G_{3}=\overline{G_{2}}$. Thus,

$$
\left(\begin{array}{c}
a_{x}(t) \\
a_{x}^{\dagger}(t)
\end{array}\right)=\left(\begin{array}{ll}
G_{1} & \frac{G_{2}}{G_{2}} \\
G_{1}
\end{array}\right)\left(\begin{array}{c}
a_{x} \\
a_{x}^{\dagger}
\end{array}\right) .
$$

From (2.7), we can obtain the following lemma, which shows an important property of the operator $\mathcal{U}_{2}^{\dagger}(t) a_{x} \mathcal{U}_{2}(t)$.

\section{Lemma 2.1. Let}

$$
G_{2}(t, x):=\mathcal{U}_{2}^{\dagger}(t) a_{x} \mathcal{U}_{2}(t) \Omega
$$

Then, $G_{2}(t, x) \in L^{2}\left(\mathbb{R}^{3}, L^{2}\left(\mathbb{R}^{3}\right)\right)$ for any fixed $t>0$. Furthermore, if we let

$$
G_{2}(t, x, z):=\left(\mathcal{U}_{2}^{\dagger}(t) a_{x} \mathcal{U}_{2}(t) \Omega\right)(z)
$$

then we have the following bound for $G_{2}$ :

$$
\left\|G_{2}(t, x, z)\right\|_{L_{x, z}^{2}} \leq C e^{K t}
$$

Proof. We first observe that

$$
\int d x\left\langle a_{x}(t) \Omega, a_{x}(t) \Omega\right\rangle=\int d x\left\langle G_{2}(t, x), G_{2}(t, x)\right\rangle .
$$

On the other hand, we have from the definition and from Lemma 6.2 that

$$
\int d x\left\langle a_{x}(t) \Omega, a_{x}(t) \Omega\right\rangle=\int d x\left\langle a_{x} \mathcal{U}_{2}(t) \Omega, a_{x} \mathcal{U}_{2}(t) \Omega\right\rangle=\left\langle\mathcal{U}_{2}(t) \Omega, \mathcal{N} \mathcal{U}_{2}(t) \Omega\right\rangle \leq C e^{K t} .
$$


Thus, from (2.11) and (2.12), we can find that $G_{2}(t, x) \in L^{2}\left(\mathbb{R}^{3}, F_{X}\right)$ for any fixed $t>0$. In particular, $\mathcal{U}_{2}^{\dagger}(t) a_{x} \mathcal{U}_{2}(t) \Omega$ is well-defined for almost every $x$. Let $\eta^{(n)}$ be a function in $L^{2}\left(\mathbb{R}^{3 n}\right)$ with $n \geq 0$. If $n \neq 1$, then the formal calculation (2.7) shows that

$$
\left\langle\eta^{(n)}, \mathcal{U}_{2}^{\dagger}(t) a_{x} \mathcal{U}_{2}(t) \Omega\right\rangle=0 .
$$

Thus, we can see that $G_{2}(t, x) \in L^{2}\left(\mathbb{R}^{3}, L^{2}\left(\mathbb{R}^{3}\right)\right)$, which proves the first part of the lemma. Now, we rewrite (2.11) and (2.12) as

$$
\int d x\left\langle a_{x}(t) \Omega, a_{x}(t) \Omega\right\rangle=\iint d x d z\left|G_{2}(t, x, z)\right|^{2} \leq C e^{K t} .
$$

This completes the proof of the lemma.

2.2. Proof of Proposition 1.1. Using the properties of $a_{x}(t)$, we can prove Proposition 1.1 .

Proof of Proposition 1.1. Recall that

$$
\begin{aligned}
& E_{2}(t, x, y) \\
= & \frac{d_{N}^{2}}{N}\left\langle\left(W(\sqrt{N} \varphi) \mathcal{U}_{2}^{\dagger}(t) a_{y} \mathcal{U}_{2}(t) \Omega\right)^{(N-1)},\left(W(\sqrt{N} \varphi) \mathcal{U}_{2}^{\dagger}(t) a_{x} \mathcal{U}_{2}(t) \Omega\right)^{(N-1)}\right\rangle \\
& +\bar{\varphi}_{t}(y) \frac{d_{N}^{2}}{\sqrt{N}}\left\langle(W(\sqrt{N} \varphi) \Omega)^{(N-1)},\left(W(\sqrt{N} \varphi) \mathcal{U}_{2}^{\dagger}(t) a_{x} \mathcal{U}_{2}(t) \Omega\right)^{(N-1)}\right\rangle \\
& +\varphi_{t}(x) \frac{d_{N}^{2}}{\sqrt{N}}\left\langle\left(W(\sqrt{N} \varphi) \mathcal{U}_{2}^{\dagger}(t) a_{y} \mathcal{U}_{2}(t) \Omega\right)^{(N-1)},(W(\sqrt{N} \varphi) \Omega)^{(N-1)}\right\rangle .
\end{aligned}
$$

We first want to compute $\left(W(\sqrt{N} \varphi) \mathcal{U}_{2}^{\dagger}(t) a_{y} \mathcal{U}_{2}(t) \Omega\right)^{(N-1)}$ explicitly. We have already seen from Lemma 2.1 that $a_{x}(t) \Omega \in L^{2}\left(\mathbb{R}^{3}, L^{2}\left(\mathbb{R}^{3}\right)\right)$ and we write it as

$$
a_{x}(t) \Omega=\mathcal{U}_{2}^{\dagger}(t) a_{x} \mathcal{U}_{2}(t) \Omega=G_{2}(t, x) .
$$

Now, we calculate from the definition of the Weyl operator that

$$
\begin{gathered}
\left(W(\sqrt{N} \varphi) \mathcal{U}_{2}^{\dagger}(t) a_{x} \mathcal{U}_{2}(t) \Omega\right)^{(N-1)} \\
=e^{-\frac{N}{2}}\left(\exp \left(a^{\dagger}(\sqrt{N} \varphi)\right) \exp (-a(\sqrt{N} \varphi)) G_{2}(t, x)\right)^{(N-1)} \\
=e^{-\frac{N}{2}}\left(\exp \left(a^{\dagger}(\sqrt{N} \varphi)\right)\left(G_{2}(t, x)-\sqrt{N} \int d z G_{2}(t, x, z) \bar{\varphi}(z)\right)\right)^{(N-1)} \\
=e^{-\frac{N}{2}}\left(\frac{1}{(N-2) !}\left(a^{\dagger}(\sqrt{N} \varphi)\right)^{N-2} G_{2}(t, x)\right. \\
\left.\quad-\frac{\sqrt{N}}{(N-1) !} \int d z G_{2}(t, x, z) \bar{\varphi}(z)\left(a^{\dagger}(\sqrt{N} \varphi)\right)^{N-1} \Omega\right)
\end{gathered}
$$

where we used the notation $G_{2}(t, x, z)$, which was defined in (2.9). Thus,

$$
\begin{aligned}
& \left(W(\sqrt{N} \varphi) \mathcal{U}_{2}^{\dagger}(t) a_{x} \mathcal{U}_{2}(t) \Omega\right)^{(N-1)}\left(x_{1}, x_{2}, \cdots, x_{N-1}\right) \\
& =\frac{1}{d_{N} \sqrt{N}}\left(\sum_{i=1}^{N-1} G_{2}\left(t, x, x_{i}\right) \prod_{j \neq i}^{N-1} \varphi\left(x_{j}\right)-N \int d z G_{2}(t, x, z) \bar{\varphi}(z) \prod_{j=1}^{N-1} \varphi\left(x_{j}\right)\right) .
\end{aligned}
$$

We also know that

$$
(W(\sqrt{N} \varphi) \Omega)^{(N-1)}\left(x_{1}, \cdots, x_{N-1}\right)=\frac{1}{d_{N}} \prod_{j=1}^{N-1} \varphi\left(x_{j}\right) .
$$


Using (2.17) and (2.19), we can explicitly calculate the terms in the right hand side of (2.15). The first term becomes

$$
\begin{aligned}
& \frac{d_{N}^{2}}{N}\left\langle\left(W(\sqrt{N} \varphi) \mathcal{U}_{2}^{\dagger}(t) a_{y} \mathcal{U}_{2}(t) \Omega\right)^{(N-1)},\left(W(\sqrt{N} \varphi) \mathcal{U}_{2}^{\dagger}(t) a_{x} \mathcal{U}_{2}(t) \Omega\right)^{(N-1)}\right\rangle \\
& =\frac{1}{N^{2}}\left\langle\sum_{i=1}^{N-1} G_{2}\left(t, y, x_{i}\right) \prod_{j \neq i}^{N-1} \varphi\left(x_{j}\right)-N \int d z G_{2}(t, y, z) \bar{\varphi}(z) \prod_{j=1}^{N-1} \varphi\left(x_{j}\right),\right. \\
& \left.\quad \sum_{i=1}^{N-1} G_{2}\left(t, x, x_{i}\right) \prod_{j \neq i}^{N-1} \varphi\left(x_{j}\right)-N \int d z G_{2}(t, x, z) \bar{\varphi}(z) \prod_{j=1}^{N-1} \varphi\left(x_{j}\right)\right\rangle_{L_{X_{N}}^{2}} \\
& =\frac{N-1}{N^{2}} \int d z \overline{G_{2}}(t, y, z) G_{2}(t, x, z)-\frac{N-2}{N^{2}} \int d z \overline{G_{2}}(t, y, z) \varphi(z) \int d z G_{2}(t, x, z) \bar{\varphi}(z) .
\end{aligned}
$$

Here $\langle\cdot\rangle_{L_{X}^{2}}$ denotes the inner product with respect to the variables $x_{1}, x_{2}, \cdots, x_{N}$ only. For the second term, we have

$$
\begin{aligned}
& \bar{\varphi}_{t}(y) \frac{d_{N}^{2}}{\sqrt{N}}\left\langle(W(\sqrt{N} \varphi) \Omega)^{(N-1)},\left(W(\sqrt{N} \varphi) \mathcal{U}_{2}^{\dagger}(t) a_{x} \mathcal{U}_{2}(t) \Omega\right)^{(N-1)}\right\rangle \\
& =\frac{\bar{\varphi}_{t}(y)}{N}\left\langle\prod_{j=1}^{N-1} \varphi\left(x_{j}\right), \sum_{i=1}^{N-1} G_{2}\left(t, x, x_{i}\right) \prod_{j \neq i}^{N-1} \varphi\left(x_{j}\right)-N \int d z G_{2}(t, x, z) \bar{\varphi}(z) \prod_{j=1}^{N-1} \varphi\left(x_{j}\right)\right\rangle_{L_{X_{N}}^{2}} \\
& =\frac{\bar{\varphi}_{t}(y)}{N}\left((N-1) \int d z G_{2}(t, x, z) \bar{\varphi}(z)-N \int d z G_{2}(t, x, z) \bar{\varphi}(z)\right) \\
& =-\frac{\bar{\varphi}_{t}(y)}{N} \int d z G_{2}(t, x, z) \bar{\varphi}(z) .
\end{aligned}
$$

A similar calculation shows for the third term that

$$
\begin{aligned}
& \varphi_{t}(x) \frac{d_{N}^{2}}{\sqrt{N}}\left\langle\left(W(\sqrt{N} \varphi) \mathcal{U}_{2}^{\dagger}(t) a_{y} \mathcal{U}_{2}(t) \Omega\right)^{(N-1)},(W(\sqrt{N} \varphi) \Omega)^{(N-1)}\right\rangle \\
& =-\frac{\varphi_{t}(x)}{N} \int d z \overline{G_{2}}(t, y, z) \varphi(z) .
\end{aligned}
$$

Therefore, the proposition follows from (2.20), (2.21), (2.22), and Lemma 2.1

\section{Strichartz Type Estimates for $\mathcal{U}_{2}(t)$}

In this section, we prove Proposition 1.2 using Strichartz estimates. For the standard Strichartz estimate used in this section, see Theorem 2.3.3 of [1].

3.1. Regularity from $\mathcal{U}_{2}(t)$ dynamics. We will use the notation

$$
H_{0}:=\int d x \nabla_{x} a_{x}^{\dagger} \nabla_{x} a_{x}
$$

to denote the kinetic energy.

Lemma 3.1. Let $H_{0}$ be defined in (3.1). Define an operator $\mathcal{T}$ on $\mathcal{F}_{X}$ by

$$
\mathcal{T}(t):=e^{-i t H_{0}} .
$$

Then, there exists a constant $C$ such that, for all $\psi \in \mathcal{F}_{X}$,

$$
\|\mathcal{T}(\cdot) \psi\|_{\widetilde{\mathcal{F}}}^{2} \leq C\|\psi\|^{2} .
$$


Proof. It is trivial that

$$
\left\|e^{-i t H_{0}} \psi^{(n)}\left(X_{n}\right)\right\|_{L_{t}^{\infty} L_{X_{n}}^{2}}^{2}=\left\|\psi^{(n)}\left(X_{n}\right)\right\|_{L_{X_{n}}^{2}}^{2} .
$$

For any fixed $i$ and $j, 1 \leq i<j \leq n$, let $\eta=x_{i}-x_{j}$. Applying the standard Strichartz estimate for Schrödinger operator on $\psi^{(n)}$, we obtain

$$
\begin{aligned}
& \left\|e^{-i t H_{0}} \psi^{(n)}\left(X_{n}\right)\right\|_{L_{t}^{2} L_{x_{i}-x_{j}}^{6} L_{x_{i}+x_{j}}^{2} L_{X_{n} \backslash\left\{x_{i}, x_{j}\right\}}^{2}}^{2}=\left\|e^{-2 i t \Delta_{\eta}} \psi^{(n)}\left(X_{n}\right)\right\|_{\left.L_{t}^{2} L_{\eta}^{6} L_{x_{i}+x_{j}}^{2} L_{X_{n} \backslash\left\{x_{i}, x_{j}\right\}}^{2}\right\}}^{2} \\
& \leq C\left\|\psi^{(n)}\left(X_{n}\right)\right\|_{L_{\eta}^{2} L_{x_{i}+x_{j}}^{2} L_{X_{n} \backslash\left\{x_{i}, x_{j}\right\}}^{2}}^{2}=C\left\|\psi^{(n)}\left(X_{n}\right)\right\|_{L_{X_{n}}^{2}}^{2} .
\end{aligned}
$$

Summing (3.4) and (3.5) over $n$, we get the desired lemma.

Lemma 3.2. Let $H_{0}$ be defined in (3.1). Define an operator $\Phi$ on $\mathcal{F}$ through

$$
\Phi_{\psi}\left(t, X_{n}\right)=\int_{0}^{t} d s e^{-i(t-s) H_{0}} \psi\left(s, X_{n}\right)
$$

for any $\psi \in \mathcal{F}$. Then, there exists a constant $C$ such that, for all $\psi \in \mathcal{F}$,

$$
\left\|\Phi_{\psi}\right\|_{\tilde{\mathcal{F}}} \leq C T\|\psi\|_{\mathcal{F}}
$$

Proof. To prove the desired lemma, it suffices to show that

$$
\left\|\Phi_{\psi}^{(n)}\right\|_{F_{n}} \leq C T\left\|\psi^{(n)}\right\|_{\mathcal{F}}
$$

Recall that we only consider a fixed time interval $[0, T]$. From the standard Strichartz estimate, we have

$$
\left\|\int_{0}^{t} d s e^{-i(t-s) H_{0}} \psi^{(n)}\left(s, X_{n}\right)\right\|_{L_{t}^{\infty} L_{X_{n}}^{2}} \leq C\left\|\psi^{(n)}\left(t, X_{n}\right)\right\|_{L_{t}^{1} L_{X_{n}}^{2}} .
$$

For any $g\left(t, X_{n}\right) \in L_{t}^{2} L_{x_{i}-x_{j}}^{6 / 5} L_{x_{i}+x_{j}}^{2} L_{X_{n} \backslash\left\{x_{i}, x_{j}\right\}}^{2}$, we obtain as the dual inequality of Lemma 3.1 that

$$
\left\|\int_{0}^{T} d t e^{i t H_{0}} g\left(t, X_{n}\right)\right\|_{L_{X_{n}}^{2}} \leq C\left\|g\left(t, X_{n}\right)\right\|_{L_{t}^{2} L_{x_{i}-x_{j}}^{6 / 5} L_{x_{i}+x_{j}}^{2} L_{X_{n} \backslash\left\{x_{i}, x_{j}\right\}}^{2}} .
$$

From the standard Strichartz estimate, we also have

$$
\left\|\int_{0}^{T} d s e^{i s H_{0}} \psi^{(n)}\left(s, X_{n}\right)\right\|_{L_{X_{n}}^{2}} \leq C\left\|\psi^{(n)}\left(t, X_{n}\right)\right\|_{L_{t}^{1} L_{X_{n}}^{2}} .
$$

Combining (3.10) and (3.11), we get

$$
\begin{aligned}
& \left\|\int_{0}^{T} d s e^{-i(t-s) H_{0}} \psi^{(n)}\left(s, X_{n}\right)\right\|_{L_{t}^{2} L_{x_{i}-x_{j}}^{6} L_{x_{i}+x_{j}}^{2} L_{X_{n} \backslash\left\{x_{i}, x_{j}\right\}}^{2}} \\
& =\sup _{g} \int_{0}^{T} d t \int_{0}^{T} d s\left\langle g\left(t, X_{n}\right), e^{-i(t-s) H_{0}} \psi^{(n)}\left(s, X_{n}\right)\right\rangle \\
& =\sup _{g}\left\langle\int_{0}^{T} d t e^{i t H_{0}} g\left(t, X_{n}\right), \int_{0}^{T} d s e^{i s H_{0}} \psi^{(n)}\left(s, X_{n}\right)\right\rangle \\
& \leq C \sup _{g}\left\|g\left(t, X_{n}\right)\right\|_{L_{t}^{2} L_{x_{i}-x_{j}}^{6 / 5} L_{x_{i}+x_{j}}^{2} L_{X_{n} \backslash\left\{x_{i}, x_{j}\right\}}^{2}}\left\|\psi^{(n)}\left(t, X_{n}\right)\right\|_{L_{t}^{1} L_{X_{n}}^{2}}=C\left\|\psi^{(n)}\left(t, X_{n}\right)\right\|_{L_{t}^{1} L_{X_{n}}^{2}},
\end{aligned}
$$

where the supremum is taken over all funtions $g$ satisfying

$$
\left\|g\left(t, X_{n}\right)\right\|_{L_{t}^{2} L_{x_{i}-x_{j}}^{6 / 5} L_{x_{i}+x_{j}}^{2} L_{X_{n} \backslash\left\{x_{i}, x_{j}\right\}}^{2}}=1 .
$$


Applying Christ-Kiselev lemma to (3.12), we finally obtain that

$$
\left\|\int_{0}^{t} d s e^{-i(t-s) H_{0}} \psi^{(n)}\left(s, X_{n}\right)\right\|_{L_{t}^{2} L_{x_{i}-x_{j}}^{6} L_{x_{i}+x_{j}}^{2} L_{X_{n} \backslash\left\{x_{i}, x_{j}\right\}}^{2}} \leq C\left\|\psi^{(n)}\left(t, X_{n}\right)\right\|_{L_{t}^{1} L_{X_{n}}^{2}} .
$$

Thus, it follows from (3.9) and (3.14) that

$$
\left\|\Phi_{\psi}^{(n)}\right\|_{F_{n}} \leq C\left\|\psi^{(n)}\left(t, X_{n}\right)\right\|_{L_{t}^{1} L_{X_{n}}^{2}} \leq C T\left\|\psi^{(n)}\left(t, X_{n}\right)\right\|_{L_{t}^{\infty} L_{X_{n}}^{2}},
$$

which proves the claim (3.8). This concludes the proof of the desired lemma.

3.2. Estimate on $H_{2}(t)$. To prove Proposition 1.2, we need the following lemma.

Lemma 3.3. Let $V=V_{1}+V_{2}$ with $V_{1} \in L^{3}$ and $V_{2} \in L^{\infty}$. Then, for all $j \in \mathbb{N}$, there exists a constant $C$ depending only on $\left\|V_{1}\right\|_{L^{3}},\left\|V_{2}\right\|_{L^{\infty}}$ and $\|\varphi\|_{H^{1}}$, such that

$$
\left\|\mathcal{N}^{j}\left(H_{2}(t)-H_{0}\right) \psi\right\| \leq C\left\|(\mathcal{N}+2)^{j+1} \psi\right\|
$$

for all $\psi \in \mathcal{F}_{X}$.

Proof. Let

$$
\begin{aligned}
L & :=\int d x\left(V *\left|\varphi_{t}\right|^{2}\right)(x) a_{x}^{\dagger} a_{x}, \\
M & :=\iint d x d y V(x-y) \overline{\varphi_{t}}(x) \varphi_{t}(y) a_{y}^{\dagger} a_{x}, \\
B & :=\frac{1}{2} \iint d x d y V(x-y) \overline{\varphi_{t}}(x) \overline{\varphi_{t}}(y) a_{x} a_{y} .
\end{aligned}
$$

Then, $H_{2}-H_{0}=L+M+B+B^{\dagger}$.

Note that

$$
\left\||V| *|\varphi|^{2}\right\|_{\infty} \leq\left\|V_{1}\right\|_{L^{3}}\|\varphi\|_{L^{6}}\|\varphi\|_{L^{2}}+\left\|V_{2}\right\|_{L^{\infty}}\|\varphi\|_{L^{2}}^{2} \leq C .
$$

Since $L$ commutes with $\mathcal{N}$ and $V *\left|\varphi_{t}\right|^{2} \in L^{\infty}$, there exists a constant $C$ such that

$$
\left\|\mathcal{N}^{j} L \psi\right\|=\left\|L \mathcal{N}^{j} \psi\right\| \leq C\left\|\mathcal{N}^{j+1} \psi\right\| .
$$

To prove an estimate for $\left\|\mathcal{N}^{j} M \psi\right\|=\left\|M \mathcal{N}^{j} \psi\right\|$, we observe that, for any $\xi \in \mathcal{F}_{X}$,

$$
\begin{aligned}
& \left|\left\langle\xi, M \mathcal{N}^{j} \psi\right\rangle\right|=\left|\iint d x d y V(x-y) \bar{\varphi}_{t}(x) \varphi_{t}(y)\left\langle(\mathcal{N}+1)^{-1 / 2} a_{y} \xi,(\mathcal{N}+1)^{1 / 2} a_{x} \mathcal{N}^{j} \psi\right\rangle\right| \\
& \leq\left\||V| *|\varphi|^{2}\right\|_{\infty}\left(\int d y\left\|(\mathcal{N}+1)^{-1 / 2} a_{y} \xi\right\|^{2}\right)^{1 / 2}\left(\int d x\left\|(\mathcal{N}+1)^{1 / 2} a_{x} \mathcal{N}^{j} \psi\right\|^{2}\right)^{1 / 2} \\
& \leq C\|\xi\|\left\|\mathcal{N}^{j+1} \psi\right\| .
\end{aligned}
$$

Since $\xi$ was arbitrary, this shows that

$$
\left\|\mathcal{N}^{j} M \psi\right\| \leq C\left\|\mathcal{N}^{j+1} \psi\right\|
$$

Estimates for $\left\|\mathcal{N}^{j} B \psi\right\|$ and $\left\|\mathcal{N}^{j} B^{\dagger} \psi\right\|$ can be obtained similarly. Since

$$
\iint d x d y|V(x-y)|^{2}\left|\varphi_{t}(x)\right|^{2}\left|\varphi_{t}(y)\right|^{2} \leq\left\|V_{1}\right\|_{L^{3}}^{2}\left\|\varphi_{t}\right\|_{L^{6}}^{2}\left\|\varphi_{t}\right\|_{L^{2}}^{2}+\left\|V_{2}\right\|_{L^{\infty}}^{2}\left\|\varphi_{t}\right\|_{L^{2}}^{2}\left\|\varphi_{t}\right\|_{L^{2}}^{2} \leq C,
$$


we have that, for any $\xi \in \mathcal{F}_{X}$,

$$
\begin{aligned}
& \left|\left\langle\xi, \mathcal{N}^{j} B \psi\right\rangle\right|=\left|\iint d x d y V(x-y) \bar{\varphi}_{t}(x) \varphi_{t}(y)\left\langle\xi, a_{x} a_{y}(\mathcal{N}-2)^{j} \psi\right\rangle\right| \\
& \leq\left(\iint d x d y|V(x-y)|^{2}\left|\varphi_{t}(x)\right|^{2}\left|\varphi_{t}(y)\right|^{2}\|\xi\|^{2}\right)^{1 / 2}\left(\int d x d y\left\|a_{x} a_{y}(\mathcal{N}-2)^{j} \psi\right\|^{2}\right)^{1 / 2} \\
& \leq C\|\xi\|\left\|\mathcal{N}^{j+2} \psi\right\| .
\end{aligned}
$$

Again, since $\xi$ was arbitrary, this shows that

$$
\left\|\mathcal{N}^{j} B \psi\right\| \leq C\left\|\mathcal{N}^{j+1} \psi\right\|
$$

Similarly, we also have that

$$
\left\|\mathcal{N}^{j} B^{\dagger} \psi\right\| \leq C\left\|(\mathcal{N}+2)^{j+1} \psi\right\|
$$

Thus, from (3.21), (3.23), (3.26), and (3.27), we get

$$
\left\|\mathcal{N}^{j}\left(H_{2}(t)-H_{0}\right) \psi\right\| \leq\left\|\mathcal{N}^{j} L \psi\right\|+\left\|\mathcal{N}^{j} M \psi\right\|+\left\|\mathcal{N}^{j}\left(B+B^{\dagger}\right) \psi\right\| \leq C\left\|(N+2)^{j+1} \psi\right\|,
$$

which proves the desired lemma.

3.3. Proof of Proposition 1.2. From Lemma 3.1, Lemma 3.2, and Lemma 3.3, we can prove Proposition 1.2

Proof of Proposition 1.2, By using the group $e^{-i t H_{0}}$ generated by $H_{0}$, we can write the evolution of $\mathcal{N}^{j} \psi_{2}(t)$ as

$$
\mathcal{N}^{j} \psi_{2}(t)=e^{-i t H_{0}} \mathcal{N}^{j} \psi+\int_{s}^{t} d \sigma e^{-i(t-\sigma) H_{0}} \mathcal{N}^{j}\left(H_{2}(\sigma)-H_{0}\right) \psi_{2}(\sigma) .
$$

Thus, by Lemma 3.1, Lemma 3.2, and Lemma 3.3, we have

$$
\begin{aligned}
\left\|\mathcal{N}^{j} \psi_{2}\right\|_{\tilde{\mathcal{F}}} & \leq C\left\|\mathcal{N}^{j} \psi\right\|+C T\left\|\mathcal{N}^{j}\left(H_{2}(\cdot)-H_{0}\right) \psi_{2}(\cdot)\right\|_{\mathcal{F}} \\
& \leq C\left\|\mathcal{N}^{j} \psi\right\|+C T\left\|(\mathcal{N}+2)^{j+1} \psi_{2}\right\|_{\mathcal{F}}
\end{aligned}
$$

which was to be proved.

\section{Difference between $\mathcal{U}(t)$ Dynamics and $\mathcal{U}_{2}(t)$ Dynamics}

4.1. Estimates on $H_{3}(t)$ and $H_{4}$. From the regularity we have seen in Proposition 1.2, we can obtain the following estimates.

Lemma 4.1. Let $V=V_{1}+V_{2}$ with $V_{1} \in L^{3}$ and $V_{2} \in L^{\infty}$. Then, for all $j \in \mathbb{N}$, there exists a constant $C$ depending only on $j,\left\|V_{1}\right\|_{L^{3}},\left\|V_{2}\right\|_{L^{\infty}}$ and $\|\varphi\|_{H^{1}}$, such that

$$
\left\|\mathcal{N}^{j} H_{3}(t) \psi\right\| \leq \frac{C}{\sqrt{N}}\left\|(\mathcal{N}+1)^{j+\frac{3}{2}} \psi\right\|
$$

for all $\psi \in \mathcal{F}_{X}$.

Proof. Let

$$
A_{3}(t)=\iint d x d y V(x-y) \bar{\varphi}_{t}(y) a_{x}^{\dagger} a_{y} a_{x}
$$

Then,

$$
\mathcal{N}^{j} H_{3}(t)=\frac{1}{\sqrt{N}}\left(\mathcal{N}^{j} A_{3}^{\dagger}(t)+\mathcal{N}^{j} A_{3}(t)\right)
$$


Now we estimate $\mathcal{N}^{j} A_{3}^{\dagger}(t)$ and $\mathcal{N}^{j} A_{3}(t)$ separately. The first term $\mathcal{N}^{j} A_{3}^{\dagger}(t)$ satisfies

$$
\begin{aligned}
& \left\|\mathcal{N}^{j} A_{3}^{\dagger}(t) \psi\left(X_{n}\right)\right\|_{L_{X_{n}}^{2}}^{2} \\
& =\sum_{n=3}^{\infty} n^{j+1}(n-1)^{2} \int d x_{3} \cdots d x_{n} \int d x_{1} d x_{2}\left|V\left(x_{1}-x_{2}\right)\right|^{2}\left|\varphi_{t}\left(x_{2}\right)\right|^{2}\left|\psi^{(n-1)}\left(x_{1}, x_{3}, \cdots, x_{n}\right)\right|^{2} \\
& \quad+2^{j+1} \int d x_{1} d x_{2}\left|V\left(x_{1}-x_{2}\right)\right|^{2}\left|\varphi_{t}\left(x_{2}\right)\right|^{2}\left|\psi^{(1)}\left(x_{1}\right)\right|^{2} \\
& \leq 2 \sum_{n=3}^{\infty} n^{j+1}(n-1)^{2} \\
& \quad \times \int d x_{3} \cdots d x_{n}\left(\left\|V_{1}\right\|_{L^{3}}^{2}\left\|\varphi_{t}\right\|_{L^{6}}^{2}+\left\|V_{2}\right\|_{L^{\infty}}^{2}\left\|\varphi_{t}\right\|_{L^{2}}^{2}\right)\left\|\psi^{(n-1)}\left(x_{1}, x_{3}, \cdots, x_{n}\right)\right\|_{L_{x_{1}}^{2}}^{2} \\
& \quad+2^{j+2}\left(\left\|V_{1}\right\|_{L^{3}}^{2}\left\|\varphi_{t}\right\|_{L^{6}}^{2}+\left\|V_{2}\right\|_{L^{\infty}}^{2}\left\|\varphi_{t}\right\|_{L^{2}}^{2}\right)\left\|\psi^{(1)}\left(x_{1}\right)\right\|_{L_{x_{1}}^{2}}^{2} \\
& \leq C
\end{aligned}
$$

and $\mathcal{N}^{j} A_{3}(t)$ satisfies

$$
\begin{aligned}
& \left\|\mathcal{N}^{j} A_{3}(t) \psi\left(X_{n}\right)\right\|_{L_{X_{n}}^{2}}^{2} \\
& =\sum_{n=1}^{\infty} n^{j+2}(n+1) \int d x_{1} \cdots d x_{n}\left|\int d y V\left(x_{1}-y\right) \varphi_{t}(y) \psi^{(n+1)}\left(y, x_{1}, \cdots, x_{n}\right)\right|^{2} \\
& \leq 2 \sum_{n=1}^{\infty} n^{j+2}(n+1) \int d x_{1} \cdots d x_{n}\left(\left\|V_{1}\right\|_{L^{3}}^{2}\left\|\varphi_{t}\right\|_{L^{6}}^{2}+\left\|V_{2}\right\|_{L^{\infty}}^{2}\left\|\varphi_{t}\right\|_{L^{2}}^{2}\right)\left\|\psi^{(n+1)}\left(y, x_{1}, \cdots, x_{n}\right)\right\|_{L_{y}^{2}}^{2} \\
& \leq C\left\|\mathcal{N}^{j+\frac{3}{2}} \psi\right\|^{2} .
\end{aligned}
$$

Hence, from (4.3), (4.4), and (4.5) we get

$$
\left\|\mathcal{N}^{j} H_{3}(t) \psi\right\| \leq \frac{C}{\sqrt{N}}\left\|(\mathcal{N}+1)^{j+\frac{3}{2}} \psi\right\|
$$

which was to be proved.

Lemma 4.2. Let $V=V_{1}+V_{2}$ with $V_{1} \in L^{3}$ and $V_{2} \in L^{\infty}$. Then, for all $j \in \mathbb{N}$, there exists a constant $C$ depending only on $j,\left\|V_{1}\right\|_{L^{3}},\left\|V_{2}\right\|_{L^{\infty}}$ and $\|\varphi\|_{H^{1}}$, such that

$$
\int_{0}^{t} d s\left\|\mathcal{N}^{j} H_{4} \mathcal{U}_{2}\left(s ; s_{1}\right) \psi\right\|^{2} \leq \frac{C}{N^{2}}\left\|\mathcal{N}^{j+2} \psi\right\|^{2}+\frac{C T}{N^{2}}\left\|(\mathcal{N}+2)^{j+3} \mathcal{U}_{2}\left(\cdot ; s_{1}\right) \psi\right\|_{\mathcal{F}}^{2}
$$

for all $\psi \in \mathcal{F}_{X}$. 
Proof. From Hölder's inequality, we have

$$
\begin{aligned}
& \left\|\mathcal{N}^{j} H_{4} \mathcal{U}_{2}\left(s ; s_{1}\right) \psi\right\|^{2}=\frac{1}{N^{2}} \sum_{n=2}^{\infty} n^{2 j} \int d X_{n}\left|\sum_{i<j}^{n} V\left(x_{i}-x_{j}\right)\left(\mathcal{U}_{2}\left(s ; s_{1}\right) \psi\right)^{(n)}\left(X_{n}\right)\right|^{2} \\
& \leq \frac{1}{N^{2}} \sum_{n=2}^{\infty} n^{2 j} \frac{n(n-1)}{2} \sum_{i<j}^{n} \int d X_{n}\left|V\left(x_{i}-x_{j}\right)\left(\mathcal{U}_{2}\left(s ; s_{1}\right) \psi\right)^{(n)}\left(X_{n}\right)\right|^{2} \\
& \leq \frac{C}{N^{2}} \sum_{n=2}^{\infty} n^{2 j+2} \sum_{i<j}^{n} \int d\left(x_{i}-x_{j}\right)\left|V\left(x_{i}-x_{j}\right)\right|^{2} \\
& \quad \times \int d\left(x_{i}+x_{j}\right) d x_{1} \cdots \widehat{d x_{i}} \cdots \widehat{d x_{j}} \cdots d x_{n}\left|\left(\mathcal{U}_{2}\left(s ; s_{1}\right) \psi\right)^{(n)}\left(x_{1}, \cdots, x_{n}\right)\right|^{2} \\
& \leq \frac{C}{N^{2}} \sum_{n=2}^{\infty} n^{2 j+2} \sum_{i<j}^{n}\left(\left\|V_{1}\right\|_{L^{3}}^{2}\left\|\left(\mathcal{U}_{2}\left(s ; s_{1}\right) \psi\right)^{(n)}\left(x_{1}, \cdots, x_{n}\right)\right\|_{L_{x_{i}-x_{j}}^{6}}^{2} L_{x_{i}+x_{j}}^{2} L_{X_{n} \backslash\left\{x_{i}, x_{j}\right\}}^{2}\right. \\
& \left.\quad+\left\|V_{2}\right\|_{L^{\infty}}^{2}\left\|\left(\mathcal{U}_{2}\left(s ; s_{1}\right) \psi\right)^{(n)}\left(x_{1}, \cdots, x_{n}\right)\right\|_{L_{X_{n}}^{2}}^{2}\right) .
\end{aligned}
$$

Thus,

$$
\int_{0}^{t} d s\left\|\mathcal{N}^{j} H_{4} \mathcal{U}_{2}\left(s ; s_{1}\right) \psi\right\|^{2} \leq \frac{C}{N^{2}}\left\|\mathcal{N}^{j+2} \mathcal{U}_{2}\left(\cdot ; s_{1}\right) \psi\right\|_{\widetilde{\mathcal{F}}}^{2}+\frac{C T}{N^{2}}\left\|\mathcal{N}^{j+2} \mathcal{U}_{2}\left(\cdot ; s_{1}\right) \psi\right\|_{\mathcal{F}}^{2}
$$

Proposition 1.2 shows that

$$
\left\|\mathcal{N}^{j+2} \mathcal{U}_{2}\left(\cdot ; s_{1}\right) \psi\right\|_{\widetilde{\mathcal{F}}} \leq C\left\|\mathcal{N}^{j+2} \psi\right\|+C T\left\|(\mathcal{N}+2)^{j+2} \mathcal{U}_{2}\left(\cdot ; s_{1}\right) \psi\right\|_{\mathcal{F}}
$$

Therefore,

$$
\int_{0}^{t} d s\left\|\mathcal{N}^{j} H_{4} \mathcal{U}_{2}\left(s ; s_{1}\right) \psi\right\|^{2} \leq \frac{C}{N^{2}}\left\|\mathcal{N}^{j+2} \psi\right\|^{2}+\frac{C T}{N^{2}}\left\|(\mathcal{N}+2)^{j+3} \mathcal{U}_{2}\left(\cdot ; s_{1}\right) \psi\right\|_{\mathcal{F}}^{2},
$$

which was to be proved.

4.2. Proof of Proposition 1.3. We are ready to estimate the difference between $\mathcal{U}^{\dagger}(t) a_{y} \mathcal{U}(t) \Omega$ and $\mathcal{U}_{2}^{\dagger}(t) a_{y} \mathcal{U}_{2}(t) \Omega$ by proving Proposition 1.3 Note that we are free to use the Duhamel formula with the operators $\mathcal{U}$ and $\mathcal{U}_{2}$ on a dense subset $\mathcal{D}\left(H_{0}\right) \cap \mathcal{D}(\mathcal{N})$ of $\mathcal{F}_{X}$, which can be seen from equations (1.43) and (1.51) together with the fact that the Weyl operator $W\left(\sqrt{N} \varphi_{s}\right)$ maps $\mathcal{D}\left(H_{0}\right)$ onto $\mathcal{D}\left(H_{0}\right)$ if $\varphi_{s} \in H^{1}\left(\mathbb{R}^{3}\right)$. (See also Lemma 3.1 of [4].)

Proof. Let

$$
\mathcal{R}_{y}^{1}(t):=\left(\mathcal{U}^{\dagger}(t)-\mathcal{U}_{2}^{\dagger}(t)\right) a_{y} \mathcal{U}(t)
$$

and

$$
\mathcal{R}_{y}^{2}(t):=\mathcal{U}_{2}^{\dagger}(t) a_{y}\left(\mathcal{U}(t)-\mathcal{U}_{2}(t)\right)
$$

Then, $\mathcal{R}_{y}(t)=\mathcal{R}_{y}^{1}(t)+\mathcal{R}_{y}^{2}(t)$.

Since

$$
\mathcal{U}^{\dagger}(t)-\mathcal{U}_{2}^{\dagger}(t)=i \int_{0}^{t} d s \mathcal{U}^{\dagger}(s ; 0)\left(H_{3}(s)+H_{4}\right) \mathcal{U}_{2}^{\dagger}(t ; s)
$$



applying $\mathcal{R}_{y}^{1}(t)$ on the vacuum gives

$$
\begin{aligned}
& \left\|(\mathcal{N}+1)^{\frac{j}{2}} \mathcal{R}_{y}^{1}(t) \Omega\right\|=\left\|(\mathcal{N}+1)^{\frac{j}{2}}\left(\mathcal{U}^{\dagger}(t)-\mathcal{U}_{2}^{\dagger}(t)\right) a_{y} \mathcal{U}(t) \Omega\right\| \\
& =\left\|\int_{0}^{t} d s(\mathcal{N}+1)^{\frac{j}{2}} \mathcal{U}^{\dagger}(s)\left(H_{3}(s)+H_{4}\right) \mathcal{U}_{2}(s ; t) a_{y} \mathcal{U}(t) \Omega\right\| \\
& \leq \int_{0}^{t} d s\left\|(\mathcal{N}+1)^{\frac{j}{2}} \mathcal{U}^{\dagger}(s)\left(H_{3}(s)+H_{4}\right) \mathcal{U}_{2}(s ; t) a_{y} \mathcal{U}(t) \Omega\right\| \\
& =\int_{0}^{t} d s\left\langle\mathcal{U}^{\dagger}(s)\left(H_{3}(s)+H_{4}\right) \mathcal{U}_{2}(s ; t) a_{y} \mathcal{U}(t) \Omega,(\mathcal{N}+1)^{j} \mathcal{U}^{\dagger}(s)\left(H_{3}(s)+H_{4}\right) \mathcal{U}_{2}(s ; t) a_{y} \mathcal{U}(t) \Omega\right\rangle^{\frac{1}{2}}
\end{aligned}
$$

Thus, from Lemma 6.1 with Schwarz inequality, we obtain that

$$
\begin{aligned}
& \int d y\left\|(\mathcal{N}+1)^{\frac{j}{2}} \mathcal{R}_{y}^{1}(t) \Omega\right\|^{2} \\
& \leq C t \int d y \int_{0}^{t} d s\left\|(\mathcal{N}+1)^{j+1} H_{3}(s) \mathcal{U}_{2}(s ; t) a_{y} \mathcal{U}(t) \Omega\right\|^{2} \\
& \quad+C t \int d y \int_{0}^{t} d s\left\|(\mathcal{N}+1)^{j+1} H_{4} \mathcal{U}_{2}(s ; t) a_{y} \mathcal{U}(t) \Omega\right\|^{2} .
\end{aligned}
$$

Now, from Lemma 6.1 Lemma 6.2, and Lemma 4.1, the first term in the right hand side of (4.16) can be estimated as

$$
\begin{aligned}
& \int d y \int_{0}^{t} d s\left\|(\mathcal{N}+1)^{j+1} H_{3}(s) \mathcal{U}_{2}(s ; t) a_{y} \mathcal{U}(t) \Omega\right\|^{2} \\
& \leq \frac{C}{N} \int d y \int_{0}^{t} d s\left\|(\mathcal{N}+1)^{j+\frac{5}{2}} \mathcal{U}_{2}(s ; t) a_{y} \mathcal{U}(t) \Omega\right\|^{2} \\
& \leq \frac{C e^{K T}}{N} \int d y \int_{0}^{t} d s\left\langle a_{y} \mathcal{U}(t) \Omega,(\mathcal{N}+1)^{2 j+5} a_{y} \mathcal{U}(t) \Omega\right\rangle \\
& \leq \frac{C e^{K T}}{N} \int_{0}^{t} d s\left\langle\mathcal{U}(t) \Omega,(\mathcal{N}+1)^{2 j+6} \mathcal{U}(t) \Omega\right\rangle \leq \frac{C e^{K T}}{N} \int_{0}^{t} d s\left\langle\Omega,(\mathcal{N}+1)^{4 j+14} \Omega\right\rangle \\
& \leq \frac{C e^{K T}}{N} .
\end{aligned}
$$

Similarly, from Lemma 6.1. Lemma 6.2, and Lemma 4.2 the second term in the right hand side of (4.16) can be estimated as

$$
\begin{aligned}
& \int d y \int_{0}^{t} d s\left\|(\mathcal{N}+1)^{j+1} H_{4} \mathcal{U}_{2}(s ; t) a_{y} \mathcal{U}(t) \Omega\right\|^{2} \\
& \leq \frac{C}{N^{2}} \int d y\left\|(\mathcal{N}+1)^{j+3} a_{y} \mathcal{U}(t) \Omega\right\|^{2}+\frac{C T}{N^{2}} \int d y\left\|(\mathcal{N}+1)^{j+4} \mathcal{U}_{2}(\cdot ; t) a_{y} \mathcal{U}(t) \Omega\right\|_{\mathcal{F}}^{2} \\
& \leq \frac{C}{N^{2}}\left\langle\mathcal{U}(t) \Omega, \mathcal{N}^{2 j+7} \mathcal{U}(t) \Omega\right\rangle+\frac{C e^{K T}}{N^{2}}\left\langle\mathcal{U}(t) \Omega, \mathcal{N}^{2 j+9} \mathcal{U}(t) \Omega\right\rangle \\
& \leq \frac{C e^{K T}}{N^{2}}\left\langle\Omega,(\mathcal{N}+1)^{4 j+20} \Omega\right\rangle=\frac{C e^{K T}}{N^{2}}
\end{aligned}
$$

Hence, from (4.16), (4.17), and (4.18), we get

$$
\int d y\left\|(\mathcal{N}+1)^{\frac{j}{2}} \mathcal{R}_{y}^{1}(t) \Omega\right\|^{2} \leq \frac{C e^{K T}}{N} .
$$


The study of $\mathcal{R}_{y}^{2}(t)$ is similar and gives

$$
\int d y\left\|(\mathcal{N}+1)^{\frac{j}{2}} \mathcal{R}_{y}^{2}(t) \Omega\right\|^{2} \leq \frac{C e^{K T}}{N} .
$$

Therefore,

$$
\left(\int d y\left\|(\mathcal{N}+1)^{\frac{j}{2}} \mathcal{R}_{y}(t) \Omega\right\|^{2}\right)^{\frac{1}{2}} \leq \frac{C e^{K T}}{\sqrt{N}},
$$

which was to be proved.

\section{Proof of Main Theorem}

Now, we are ready to prove the main theorem.

Proof of Main Theorem. First, we write the difference between $E(t, x, y)$ and $E_{2}(t, x, y)$ in detail as follows.

$$
\begin{aligned}
E & (t, x, y)-E_{2}(t, x, y) \\
= & \frac{d_{N}^{2}}{N}\left\langle\left(W(\sqrt{N} \varphi) \mathcal{R}_{y}(t) \Omega\right)^{(N-1)},\left(W(\sqrt{N} \varphi) \mathcal{U}_{2}^{\dagger}(t) a_{x} \mathcal{U}_{2}(t) \Omega\right)^{(N-1)}\right\rangle \\
& +\frac{d_{N}^{2}}{N}\left\langle\left(W(\sqrt{N} \varphi) \mathcal{U}_{2}^{\dagger}(t) a_{y} \mathcal{U}_{2}(t) \Omega\right)^{(N-1)},\left(W(\sqrt{N} \varphi) \mathcal{R}_{x}(t) \Omega\right)^{(N-1)}\right\rangle \\
& +\frac{d_{N}^{2}}{N}\left\langle\left(W(\sqrt{N} \varphi) \mathcal{R}_{y}(t) \Omega\right)^{(N-1)},\left(W(\sqrt{N} \varphi) \mathcal{R}_{x}(t) \Omega\right)^{(N-1)}\right\rangle \\
& +\bar{\varphi}_{t}(y) \frac{d_{N}^{2}}{\sqrt{N}}\left\langle(W(\sqrt{N} \varphi) \Omega)^{(N-1)},\left(W(\sqrt{N} \varphi) \mathcal{R}_{x}(t) \Omega\right)^{(N-1)}\right\rangle \\
& +\varphi_{t}(x) \frac{d_{N}^{2}}{\sqrt{N}}\left\langle\left(W(\sqrt{N} \varphi) \mathcal{R}_{y}(t) \Omega\right)^{(N-1)},(W(\sqrt{N} \varphi) \Omega)^{(N-1)}\right\rangle .
\end{aligned}
$$

From Lemma 6.2 and Proposition 1.3, the first term in the right hand side of (5.1) can be estimate as

$$
\begin{aligned}
& \frac{d_{N}^{2}}{N}\left(\int d x d y\left|\left\langle\left(W(\sqrt{N} \varphi) \mathcal{R}_{y}(t) \Omega\right)^{(N-1)},\left(W(\sqrt{N} \varphi) \mathcal{U}_{2}^{\dagger}(t) a_{x} \mathcal{U}_{2}(t) \Omega\right)^{(N-1)}\right\rangle\right|^{2}\right)^{\frac{1}{2}} \\
& \leq \frac{d_{N}^{2}}{N}\left(\int d y\left\|\mathcal{R}_{y}(t) \Omega\right\|^{2}\right)^{\frac{1}{2}}\left(\int d x\left\|\mathcal{U}_{2}^{\dagger}(t) a_{x} \mathcal{U}_{2}(t) \Omega\right\|^{2}\right)^{\frac{1}{2}} \\
& \leq \frac{d_{N}^{2}}{N} \cdot \frac{C e^{K T}}{\sqrt{N}} \cdot C e^{K T} \leq \frac{C e^{K T}}{N}
\end{aligned}
$$

Similarly, the second term can be estimated as

$$
\frac{d_{N}^{2}}{N}\left(\int d x d y\left|\left\langle\left(W(\sqrt{N} \varphi) \mathcal{U}_{2}^{\dagger}(t) a_{y} \mathcal{U}_{2}(t) \Omega\right)^{(N-1)},\left(W(\sqrt{N} \varphi) \mathcal{R}_{x}(t) \Omega\right)^{(N-1)}\right\rangle\right|^{2}\right)^{\frac{1}{2}} \leq \frac{C e^{K T}}{N} .
$$

To estimate the third term, we use the following estimate

$$
\begin{aligned}
& \frac{d_{N}^{2}}{N}\left(\int d x d y\left|\left\langle\left(W(\sqrt{N} \varphi) \mathcal{R}_{y}(t) \Omega\right)^{(N-1)},\left(W(\sqrt{N} \varphi) \mathcal{R}_{x}(t) \Omega\right)^{(N-1)}\right\rangle\right|^{2}\right)^{\frac{1}{2}} \\
& \leq \frac{d_{N}^{2}}{N}\left(\int d y\left\|\mathcal{R}_{y}(t) \Omega\right\|^{2}\right)^{\frac{1}{2}}\left(\int d x\left\|\mathcal{R}_{x}(t) \Omega\right\|^{2}\right)^{\frac{1}{2}} \leq \frac{C e^{K T}}{N \sqrt{N}}
\end{aligned}
$$


To estimate the fourth term in the right hand side of (5.1), we first note that

$$
\begin{aligned}
& \left\langle(W(\sqrt{N} \varphi) \Omega)^{(N-1)},\left(W(\sqrt{N} \varphi) \mathcal{R}_{x}(t) \Omega\right)^{(N-1)}\right\rangle \\
& =\frac{1}{d_{N}}\left\langle\varphi^{\otimes(N-1)},\left(W(\sqrt{N} \varphi) \mathcal{R}_{x}(t) \Omega\right)^{(N-1)}\right\rangle \\
& =\frac{1}{d_{N}}\left\langle\varphi^{\otimes(N-1)}, W(\sqrt{N} \varphi) \mathcal{R}_{x}(t) \Omega\right\rangle=\frac{1}{d_{N}}\left\langle W(\sqrt{N} \varphi)^{\dagger} \varphi^{\otimes(N-1)}, \mathcal{R}_{x}(t) \Omega\right\rangle \\
& =\frac{1}{d_{N}}\left\langle(\mathcal{N}+1)^{-\frac{1}{2}} W(\sqrt{N} \varphi)^{\dagger} \varphi^{\otimes(N-1)},(\mathcal{N}+1)^{\frac{1}{2}} \mathcal{R}_{x}(t) \Omega\right\rangle .
\end{aligned}
$$

It follows from Proposition 1.3 and Lemma 6.3 that

$$
\begin{aligned}
& \left(\int d x\left|\left\langle(\mathcal{N}+1)^{-\frac{1}{2}} W(\sqrt{N} \varphi)^{\dagger} \varphi^{\otimes(N-1)},(\mathcal{N}+1)^{\frac{1}{2}} \mathcal{R}_{x}(t) \Omega\right\rangle\right|^{2}\right)^{\frac{1}{2}} \\
& \leq\left\|(\mathcal{N}+1)^{-\frac{1}{2}} W(\sqrt{N} \varphi)^{\dagger} \varphi^{\otimes(N-1)}\right\|\left(\int d x\left\|(\mathcal{N}+1)^{\frac{1}{2}} \mathcal{R}_{x}(t) \Omega\right\|^{2}\right)^{\frac{1}{2}} \\
& \leq \frac{C e^{K T}}{d_{N} \sqrt{N}} .
\end{aligned}
$$

Thus, from (5.5) and (5.6), we obtain

$$
\begin{aligned}
& \left(\int d x d y\left|\overline{\varphi_{t}}(y) \frac{d_{N}^{2}}{\sqrt{N}}\left\langle(W(\sqrt{N} \varphi) \Omega)^{(N-1)},\left(W(\sqrt{N} \varphi) \mathcal{R}_{x}(t) \Omega\right)^{(N-1)}\right\rangle\right|^{2}\right)^{\frac{1}{2}} \\
& \leq \frac{C e^{K T}}{N} .
\end{aligned}
$$

The last term in the right hand side of (5.1) can also be similarly estimated as

$$
\begin{aligned}
& \left(\int d x d y\left|\varphi_{t}(x) \frac{d_{N}^{2}}{\sqrt{N}}\left\langle\left(W(\sqrt{N} \varphi) \mathcal{R}_{y}(t) \Omega\right)^{(N-1)},(W(\sqrt{N} \varphi) \Omega)^{(N-1)}\right\rangle\right|^{2}\right)^{\frac{1}{2}} \\
& \leq \frac{C e^{K T}}{N} .
\end{aligned}
$$

Therefore, together with Proposition [1.1] inserting (5.2), (5.3), (5.4), (5.7), and, (5.8) into (5.1) yields

$$
\|E(t, x, y)\|_{L_{x, y}^{2}} \leq\left\|E_{2}(t, x, y)\right\|_{L_{x, y}^{2}}+\frac{C e^{K T}}{N} \leq \frac{C e^{K T}}{N} .
$$

By Remark 2 and Remark 3 , this completes the proof of the main theorem.

\section{Expectation of Number Operator with respect to the Various Evolutions}

The following lemma shows that the expectation of the number operator with respect to the full evolution is bounded uniformly in $N$.

Lemma 6.1 (Proposition 3.3 of [10]). Suppose that $V=V_{1}+V_{2}$ where $V_{1} \in L^{3}\left(\mathbb{R}^{3}\right)$ and $V_{2} \in L^{\infty}\left(\mathbb{R}^{3}\right)$. Let $\mathcal{U}(t ; s)$ be the operator satisfying (1.43). Then, for all $j \in \mathbb{N}$, there exist constants $C$ and $K$, depending only on $j,\|\varphi\|_{H^{1}}$, and $\|V\|_{L_{3}}$ such that

$$
\left\langle\mathcal{U}(t ; s) \psi, \mathcal{N}^{j} \mathcal{U}(t ; s) \psi\right\rangle \leq C e^{K|t-s|}\left\langle\psi,(\mathcal{N}+1)^{2 j+2} \psi\right\rangle,
$$

for all $\psi \in \mathcal{F}_{X}$. 
Remark 5. Specially, if $\psi=\Omega$, we have

$$
\left\langle\mathcal{U}(t ; s) \Omega, \mathcal{N}^{j} \mathcal{U}(t ; s) \Omega\right\rangle \leq C e^{K|t-s|} .
$$

A similar result holds for $\mathcal{U}_{2}(t)$ evolution. For completeness, we prove it here.

Lemma 6.2. Suppose that $V=V_{1}+V_{2}$ where $V_{1} \in L^{3}\left(\mathbb{R}^{3}\right)$ and $V_{2} \in L^{\infty}\left(\mathbb{R}^{3}\right)$. Let $\mathcal{U}_{2}(t ; s)$ be the operator satisfying (1.51). Then, for all $j \in \mathbb{N}$, there exist constants $C$ and $K$, depending only on $j$, $\|\varphi\|_{H^{1}}$, and $\|V\|_{L_{3}}$ such that

$$
\left\langle\mathcal{U}_{2}(t ; s) \psi, \mathcal{N}^{j} \mathcal{U}_{2}(t ; s) \psi\right\rangle \leq C e^{K|t-s|}\left\langle\psi,(\mathcal{N}+1)^{j} \psi\right\rangle,
$$

for all $\psi \in \mathcal{F}_{X}$.

Proof. It suffices to prove the lemma when $s=0$. We have

$$
\begin{aligned}
& \frac{d}{d t}\left\langle\mathcal{U}_{2}(t ; 0) \psi,(\mathcal{N}+1)^{j} \mathcal{U}_{2}(t ; 0) \psi\right\rangle=\left\langle\mathcal{U}_{2}(t ; 0) \psi,\left[i H_{2},(\mathcal{N}+1)^{j}\right] \mathcal{U}_{2}(t ; 0) \psi\right\rangle \\
& =\operatorname{Im} \iint d x d y V(x-y) \varphi_{t}(x) \varphi_{t}(y)\left\langle\mathcal{U}_{2}(t ; 0) \psi,\left[a_{x}^{\dagger} a_{y}^{\dagger},(\mathcal{N}+1)^{j}\right] \mathcal{U}_{2}(t ; 0) \psi\right\rangle \\
& =\operatorname{Im} \sum_{k=0}^{j-1}\left(\begin{array}{l}
j \\
k
\end{array}\right)(-1)^{k} \iint d x d y V(x-y) \varphi_{t}(x) \varphi_{t}(y) \\
& \times\left(\left\langle a_{x}(\mathcal{N}+1)^{\frac{k}{2}} \mathcal{U}_{2}(t ; 0) \psi, a_{y}^{\dagger}(\mathcal{N}+3)^{\frac{k}{2}} \mathcal{U}_{2}(t ; 0) \psi\right\rangle+\left\langle a_{x} \mathcal{N}^{\frac{k}{2}} \mathcal{U}_{2}(t ; 0) \psi, a_{y}^{\dagger}(\mathcal{N}+2)^{\frac{k}{2}} \mathcal{U}_{2}(t ; 0) \psi\right\rangle\right) \\
& \leq \sum_{k=0}^{j-1}\left(\begin{array}{l}
j \\
k
\end{array}\right) \sup _{x}\|V(x-\cdot) \varphi(\cdot)\|_{L^{2}}\left(\int d x\left|\varphi_{t}(x)\right|\left\|a_{x}(\mathcal{N}+1)^{\frac{k}{2}} \mathcal{U}_{2}(t ; 0) \psi\right\|\left\|(\mathcal{N}+3)^{\frac{k+1}{2}} \mathcal{U}_{2}(t ; 0) \psi\right\|\right. \\
& \left.\quad+\int d x\left|\varphi_{t}(x)\right|\left\|a_{x} \mathcal{N}^{\frac{k}{2}} \mathcal{U}_{2}(t ; 0) \psi\right\|\left\|(\mathcal{N}+3)^{\frac{k+1}{2}} \mathcal{U}_{2}(t ; 0) \psi\right\|\right) \\
& \leq C\left\|(\mathcal{N}+1)^{\frac{j}{2}} \mathcal{U}_{2}(t ; 0) \psi\right\|^{2}=C\left\langle\mathcal{U}_{2}(t ; 0) \psi,(\mathcal{N}+1)^{j} \mathcal{U}_{2}(t ; 0) \psi\right\rangle .
\end{aligned}
$$

Since $\mathcal{U}_{2}(0 ; 0)=I$, we also have

$$
\left\langle\mathcal{U}_{2}(0 ; 0) \psi,(\mathcal{N}+1)^{j} \mathcal{U}_{2}(0 ; 0) \psi\right\rangle=\left\langle\psi,(\mathcal{N}+1)^{j} \psi\right\rangle .
$$

Using (6.4) and (6.5) the conclusion follows directly from the Gronwall's lemma.

Lemma 6.3. There exists a constant $C$ such that

$$
\left\|(\mathcal{N}+1)^{-\frac{1}{2}} W(\sqrt{N} \varphi)^{\dagger}\left(\varphi^{\otimes(N-1)}\right)\right\| \leq \frac{C}{d_{N}} .
$$

Proof. It is obvious that $W(\sqrt{N} \varphi)^{\dagger}\left(\varphi^{\otimes(N-1)}\right)$ is a linear combination of tensor products of $\varphi$. We define the coefficients $\mathcal{A}_{m}$ such that

$$
\mathcal{A}_{m}:=\left\|\left(W(\sqrt{N} \varphi)^{\dagger}\left(\varphi^{\otimes(N-1)}\right)\right)^{(m)}\right\| .
$$

For $m \leq N-1$, we can explicitly calculate $\mathcal{A}_{m}$. Since

$$
\left(W(\sqrt{N} \varphi)^{\dagger}\left(\varphi^{\otimes(N-1)}\right)\right)^{(m)}=\left(e^{-N / 2} \exp \left(a^{\dagger}(-\sqrt{N} \varphi)\right) \exp (a(\sqrt{N} \varphi))\left(\varphi^{\otimes(N-1)}\right)\right)^{(m)},
$$

we have

$$
\begin{aligned}
\mathcal{A}_{m}=e^{-N / 2} \sum_{k=0}^{m} \frac{1}{k !}( & -\sqrt{N})^{k} \sqrt{m-k+1} \sqrt{m-k+2} \cdots \sqrt{m} \\
& \times \frac{1}{(N-m-1+k) !} \sqrt{N}{ }^{N-m-1+k} \sqrt{N-1} \sqrt{N-2} \cdots \sqrt{m-k+1}
\end{aligned}
$$


We want to estimate $\left|\mathcal{A}_{m}\right|$. When $m=0$, we have

$$
\mathcal{A}_{0}=e^{-N / 2} \frac{\sqrt{N}^{N-1}}{\sqrt{(N-1) !}}=\frac{1}{d_{N}}
$$

When $1 \leq m \leq N-1, \mathcal{A}_{m}$ can be simplified as follows.

$$
\begin{aligned}
& \mathcal{A}_{m}=e^{-N / 2} \sqrt{N}^{N-m-1} \sqrt{\frac{(N-1) !}{m !}} \sum_{k=0}^{m}(-1)^{k} N^{k} \frac{m !}{(m-k) ! k !(N-m-1+k) !} \\
& =e^{-N / 2} \sqrt{N}^{N-m-1} \sqrt{\frac{m !}{(N-1) !}} L_{m}^{(N-m-1)}(N),
\end{aligned}
$$

where the associated Laguerre polynomial $L_{n}^{(\alpha)}(x)$ is defined by

$$
L_{n}^{(\alpha)}(x):=\sum_{k=0}^{n}(-1)^{k} \frac{(n+\alpha) !}{k !(n-k) !(\alpha+k) !} x^{k} .
$$

We now follow the argument used in the proof of Lemma 4.2 in [10. The sharp estimate obtained by Krasikov [8] for $L_{n}^{(\alpha)}(x)$ shows that

$$
\left|L_{n}^{(\alpha)}(x)\right|<\sqrt{\frac{(n+\alpha) !}{n !}} \sqrt{\frac{x\left(s^{2}-q^{2}\right)}{r(x)}} e^{\frac{x}{2}} x^{-\frac{\alpha+1}{2}},
$$

where

$$
s=(n+\alpha+1)^{\frac{1}{2}}+n^{\frac{1}{2}}, \quad q=(n+\alpha+1)^{\frac{1}{2}}-n^{\frac{1}{2}}, \quad r(x)=\left(x-q^{2}\right)\left(s^{2}-x\right) .
$$

Thus, we obtain that, for $1 \leq m \leq N-1$,

$$
\begin{aligned}
\left|\mathcal{A}_{m}\right| & <e^{-N / 2} \sqrt{N}^{N-m-1} \sqrt{\frac{m !}{(N-1) !}} \sqrt{\frac{(N-1) !}{m !}} \sqrt{\frac{4 N \sqrt{N m}}{4 N m-m^{2}}} e^{N / 2} N^{-\frac{N-m}{2}}=\sqrt{\frac{4 \sqrt{N m}}{4 N m-m^{2}}} \\
& <C N^{-1 / 4} m^{-1 / 4} .
\end{aligned}
$$

For $m \geq N$, we only need the following bound.

$$
\sum_{m=N}^{\infty}\left|\mathcal{A}_{m}\right|^{2} \leq\left\|\left(W(\sqrt{N} \varphi)^{\dagger}\left(\varphi^{\otimes(N-1)}\right)\right)\right\|^{2} \leq 1
$$

Therefore, from (6.10), (6.15), and (6.16), we obtain

$$
\begin{aligned}
& \left\|(\mathcal{N}+1)^{-\frac{1}{2}} W(\sqrt{N} \varphi)^{\dagger}\left(\varphi^{\otimes(N-1)}\right)\right\|^{2}=\sum_{m=0}^{\infty} \frac{\left|\mathcal{A}_{m}\right|^{2}}{m+1} \\
& \leq \frac{1}{d_{N}^{2}}+C N^{-1 / 2} \sum_{m=1}^{N-1} \frac{1}{(m+1)^{3 / 2}}+\frac{C}{N} \sum_{m=N}^{\infty}\left|\mathcal{A}_{m}\right|^{2} \leq C N^{-1 / 2} .
\end{aligned}
$$

This proves the desired lemma.

\section{Stability of the Operator}

The following lemma shows that the operator $H_{2}(t)$ is stable. (See Proposition 3.4 of 7 for more details.)

Lemma 7.1. There exists a constant $C, K>0$ such that, for the operator $A_{2}(t)=H_{2}(t)+C(\mathcal{N}+1)$, we have the operator inequality $\dot{A}_{2}(t) \leq K A_{2}(t)$, where $\dot{A}_{2}(t)=(d / d t) A_{2}(t)$. 
Proof. Let

$$
\begin{aligned}
& \dot{H}_{2}(t):=\frac{d H_{2}(t)}{d t} \\
& =\iint d x d y V(x-y) \overline{\varphi_{t}(y)} \dot{\varphi}_{t}(y) a_{x}^{\dagger} a_{x}+\iint d x d y V(x-y) \overline{\varphi_{t}(x)} \dot{\varphi}_{t}(y) a_{y}^{\dagger} a_{x} \\
& \quad+\iint d x d y V(x-y) \varphi_{t}(x) \dot{\varphi}_{t}(y) a_{x}^{\dagger} a_{y}^{\dagger}+h . c .
\end{aligned}
$$

where h.c. denotes the Hermitian conjugate and $\dot{\varphi}_{t}=\partial_{t} \varphi_{t}$.

In order to control $\left\langle\psi, \dot{H}_{2}(t) \psi\right\rangle$ for $\psi \in \mathcal{F}_{X}$, we need to estimate terms such as

$$
\iint d x d y\left\langle\psi, V(x-y) \varphi_{t}(x) \dot{\varphi}_{t}(y) a_{x}^{\dagger} a_{y}^{\dagger} \psi\right\rangle .
$$

We know that $\varphi_{t}$ is the solution of the nonlinear Hartree equation and satisfies

$$
\dot{\varphi}_{t}(y)=-i\left[-\Delta \varphi_{t}(y)+\left(V *\left|\varphi_{t}\right|^{2}\right)(y) \varphi_{t}(y)\right] .
$$

Thus,

$$
\begin{aligned}
& \iint d x d y\left\langle\psi, V(x-y) \varphi_{t}(x) \dot{\varphi}_{t}(y) a_{x}^{\dagger} a_{y}^{\dagger} \psi\right\rangle \\
& =-i \iint d x d y\left\langle a_{x} a_{y} \psi, V(x-y) \varphi_{t}(x)\left[\left(-\Delta \varphi_{t}\right)(y)+\left(V *\left|\varphi_{t}\right|^{2}\right)(y) \varphi_{t}(y)\right] \psi\right\rangle .
\end{aligned}
$$

Since $\varphi_{t} \in H^{1}$ and

$$
\left\|V *\left|\varphi_{t}\right|^{2}\right\|_{\infty} \leq C
$$

we obtain that

$$
\begin{aligned}
& \left|\iint d x d y\left\langle a_{x} a_{y} \psi, V(x-y) \varphi_{t}(x)\left(V *\left|\varphi_{t}\right|^{2}\right)(y) \varphi_{t}(y) \psi\right\rangle\right| \\
& \leq \sum_{n=2}^{\infty} \sqrt{n(n-1)}\left\|\psi^{(n-2)}\right\| \cdot\left\|\psi^{(n)}\right\| \cdot\left\|V(x-y) \varphi_{t}(x)\left(V *\left|\varphi_{t}\right|^{2}\right)(y) \varphi_{t}(y)\right\|_{L_{x, y}^{2}} \\
& \leq C\langle\psi, \mathcal{N} \psi\rangle .
\end{aligned}
$$

By integrating by parts, we find that

$$
\begin{aligned}
& \iint d x d y \overline{\psi^{(n)}\left(x, y, X_{n-2}\right)} V(x-y) \varphi_{t}(x)\left(-\Delta \varphi_{t}\right)(y) \\
& =\iint d x d y\left(\overline{\left(\nabla_{y} \psi^{(n)}\right)\left(x, y, X_{n-2}\right)} V(x-y)-\overline{\psi^{(n)}\left(x, y, X_{n-2}\right)}(\nabla V)(x-y)\right) \cdot \varphi_{t}(x) \nabla \varphi_{t}(y) \\
& =\iint d x d y \overline{\left(\nabla_{y} \psi^{(n)}\right)\left(x, y, X_{n-2}\right)} \cdot V(x-y) \varphi_{t}(x) \nabla \varphi_{t}(y) \\
& +\iint d x d y \overline{\left(-\nabla_{x} \psi^{(n)}\right)\left(x, y, X_{n-2}\right)} \cdot V(x-y) \varphi_{t}(x) \nabla \varphi_{t}(y) \\
& +\iint d x d y \overline{\psi^{(n)}\left(x, y, X_{n-2}\right)} V(x-y)\left(-\nabla \varphi_{t}\right)(x) \cdot \nabla \varphi_{t}(y) .
\end{aligned}
$$


Thus, we get

$$
\begin{aligned}
& \left|\iint d x d y\left\langle a_{x} a_{y} \psi, V(x-y) \varphi_{t}(x)\left(-\Delta \varphi_{t}\right)(y) \psi\right\rangle\right| \\
& =\left|\sum_{n=2}^{\infty} \sqrt{n(n-1)} \int d x d y d X_{n-2} \overline{\psi^{(n)}\left(x, y, X_{n-2}\right)} V(x-y) \varphi_{t}(x)\left(-\Delta \varphi_{t}\right)(y) \psi^{(n-2)}\left(X_{n-2}\right)\right| \\
& \leq \sum_{n=2}^{\infty} \sqrt{n(n-1)} \\
& \quad \times\left(\left\|\left(\nabla_{y} \psi^{(n)}\right)\left(x, y, X_{n-2}\right)\right\|_{L_{x, y, X_{n-2}}^{2}}\left\|V(x-y) \varphi_{t}(x) \nabla \varphi_{t}(y)\right\|_{L_{x, y}^{2}\left\|\psi^{(n-2)}\left(X_{n-2}\right)\right\|_{L_{X_{n-2}}^{2}}}\left\|V(x-y) \varphi_{t}(x) \nabla \varphi_{t}(y)\right\|_{L_{x, y}^{2}}\left\|\psi^{(n-2)}\left(X_{n-2}\right)\right\|_{L_{X_{n-2}}^{2}}\right. \\
& \left.\quad+\left\|\left(\nabla_{x} \psi^{(n)}\right)\left(x, y, X_{n-2}\right)\right\|_{L_{x, y, X_{n-2}}^{2}}\|V\|_{L_{x, y, X_{n-2}}}\left\|\nabla \varphi_{t}(x) \nabla \varphi_{t}(y)\right\|_{L_{x, y}^{2}}\left\|\psi^{(n-2)}\left(X_{n-2}\right)\right\|_{L_{X_{n-2}}^{2}}\right) .
\end{aligned}
$$

When $V^{2} \leq D(1-\Delta)$,

$$
\begin{aligned}
& \left.\left\|V(x-y) \psi^{(n)}\left(x, y, X_{n-2}\right)\right\|_{L_{x, y, X_{n-2}}^{2}}+\left\|\psi^{(n)}\left(x, y, X_{n-2}\right)\right\|_{L_{x, y, X_{n-2}}^{2}}\right) . \\
& \leq D\left(\left\|\left(\nabla_{x} \psi^{(n)}\right)\left(x, y, X_{n-2}\right)\right\|_{L_{x, y, X_{n-2}}^{2}}+\right.
\end{aligned}
$$

Hence, from (7.8) and (7.9), we have

$$
\begin{aligned}
& \left|\iint d x d y\left\langle a_{x} a_{y} \psi, V(x-y) \varphi_{t}(x)\left(-\Delta \varphi_{t}\right)(y) \psi\right\rangle\right| \\
& \leq C \sum_{n=2}^{\infty} \sqrt{n(n-1)}\left\|\left(\nabla_{x} \psi^{(n)}\right)\left(x, y, X_{n-2}\right)\right\|_{L_{x, y, X_{n-2}}^{2}}\left\|\psi^{(n-2)}\left(X_{n-2}\right)\right\|_{L_{X_{n-2}}^{2}} \\
& \quad+C \sum_{n=2}^{\infty} \sqrt{n(n-1)}\left\|\psi^{(n)}\left(x, y, X_{n-2}\right)\right\|_{L_{x, y, X_{n-2}}^{2}}\left\|\psi^{(n-2)}\left(X_{n-2}\right)\right\|_{L_{X_{n-2}}^{2}} \\
& \leq C\left\langle\psi,\left(H_{0}+\mathcal{N}+1\right) \psi\right\rangle .
\end{aligned}
$$

Now, (7.4), (7.6), and (7.10) show that

$$
\left|\iint d x d y\left\langle\psi, V(x-y) \varphi_{t}(x) \dot{\varphi}_{t}(y) a_{x}^{\dagger} a_{y}^{\dagger} \psi\right\rangle\right| \leq C\left\langle\psi,\left(H_{0}+C(\mathcal{N}+1)\right) \psi\right\rangle .
$$

Other terms in the right hand side of (7.1) can be estimated similarly. Hence, we get

$$
\left\langle\psi, \dot{H}_{2}(t) \psi\right\rangle \leq C\left\langle\psi,\left(H_{0}+C(\mathcal{N}+1)\right) \psi\right\rangle .
$$

Furthermore, we also know that $H_{2}(t)-H_{0} \geq-C(\mathcal{N}+1)$, or

$$
H_{2}(t)+C(\mathcal{N}+1) \geq H_{0}
$$

(See Corollary 2.1 in [5.) Then, we can find constants $C_{0}$ and $C_{1}$ such that

$$
\left.\frac{d}{d t}\left(H_{2}(t)+C_{0}(\mathcal{N}+1)\right) \psi\right\rangle \leq C_{1}\left\langle\psi,\left(H_{2}(t)+C_{0}(\mathcal{N}+1)\right) \psi\right\rangle
$$

This proves the desired lemma.

\section{ACKNOWLEDGMENT}

We are grateful to H.-T. Yau and Benjamin Schlein for helpful discussions. 


\section{REFERENCES}

[1] Cazenave, T.: Semilinear Schrödinger Equations, American Mathematical Society, Providence (2003).

[2] Erdös, L., Schlein, B.: Quantum Dynamics with Mean Field Interactions: a New Approach, J. Stat. Phys. 134, 859-870 (2009).

[3] Erdös, L., Yau, H.-T.: Derivation of the Nonlinear Schrödinger Equation from a Many Body Coulom System, Adv. Theor. Math. Phys. 5, 1169-1205 (2001).

[4] Ginibre, J., Velo, G.: The Classical Field Limit of Scattering Theory for Non-Relativistic Many-Boson Systems. I, Commun. Math. Phys. 66, 37-76 (1979).

[5] Ginibre, J., Velo, G.: The Classical Field Limit of Scattering Theory for Non-Relativistic Many-Boson Systems. II., Commun. Math. Phys. 68, 45-68 (1979).

[6] Hepp, K.: The Classical Limit for Quantum Mechanical Correlation Functions, Commun. Math. Phys. 35, 265-277 (1974).

[7] Kato, T.: Linear Evolution Equations of "Hyperbolic" Type, J. Fac. Sci. Univ. Tokyo, Sec. I, 17, 241-258 (1970).

[8] Krasikov, I.: Inequalities for Laguerre polynomials, East J. Approx. 11, 257-268 (2005).

[9] Knowles, A., Pickl, P.: Mean-Field Dynamics: Singular Potentials and Rate of Convergence, Commun. Math. Phys. 298, 101-138 (2010).

[10] Rodnianski, I., Schlein, B.: Quantum Fluctuations and Rate of Convergence towards Mean Field Dynamics, Commun. Math. Phys. 291, 31-61 (2009).

[11] Spohn, H.: Kinetic Equations from Hamiltonian Dynamics, Rev. Mod. Phys. 52, 569-615 (1980).

Department of Mathematical Sciences, Tsinghua University, Beijing, 100084, People's Republic of China E-mail address: lchen@math.tsinghua.edu.cn

Department of Mathematical Sciences, Korea Advanced Institute of Science and Technology, Daejeon, 305701, RePublic of Korea

E-mail address: jioon.lee@kaist.edu 